\title{
Integrin $\alpha 9$ regulates smooth muscle cell phenotype switching and vascular remodeling
}

\author{
Manish Jain, ${ }^{1}$ Rishabh Dev, ${ }^{1}$ Prakash Doddapattar, ${ }^{1}$ Shigeyuki Kon, ${ }^{2}$ Nirav Dhanesha, ${ }^{1}$ and Anil K. Chauhan' \\ 'Division of Hematology-Oncology and Blood \& Marrow Transplantation, Department of Internal Medicine, Carver College \\ of Medicine, University of lowa, lowa City, lowa, USA. ${ }^{2}$ Department of Molecular Immunology, Faculty of Pharmaceutical \\ Sciences, Fukuyama University, Hiroshima, Japan.
}

\begin{abstract}
Excessive proliferation of vascular smooth muscle cells (SMCs) remains a significant cause of instent restenosis. Integrins, which are heterodimeric transmembrane receptors, play a crucial role in SMC biology by binding to the extracellular matrix protein with the actin cytoskeleton within the SMC. Integrin $\alpha 9$ plays an important role in cell motility and autoimmune diseases; however, its role in SMC biology and remodeling remains unclear. Herein, we demonstrate that stimulated human coronary SMCs upregulate $\alpha 9$ expression. Targeting $\alpha 9$ in stimulated human coronary SMCs, using anti-integrin $\alpha 9$ antibody, suppresses synthetic phenotype and inhibits SMC proliferation and migration. To provide definitive evidence, we generated an SMC-specific $\alpha 9$-deficient mouse strain. Genetic ablation of $\alpha 9$ in SMCs suppressed synthetic phenotype and reduced proliferation and migration in vitro. Mechanistically, suppressed synthetic phenotype and reduced proliferation were associated with decreased focal adhesion kinase/steroid receptor coactivator signaling and downstream targets, including phosphorylated ERK, p38 MAPK, glycogen synthase kinase 3及, and nuclear $\beta$-catenin, with reduced transcriptional activation of $\beta$-catenin target genes. Following vascular injury, SMC-specific $\alpha 9$-deficient mice or wild-type mice treated with murine anti-integrin $\alpha 9$ antibody exhibited reduced injury-induced neointimal hyperplasia at day 28 by limiting SMC migration and proliferation. Our findings suggest that integrin $\alpha 9$ regulates SMC biology, suggesting its potential therapeutic application in vascular remodeling.
\end{abstract}

Conflict of interest: The authors have declared that no conflict of interest exists.

Copyright: (ㄷ) 2021, Jain et al. This is an open access article published under the terms of the Creative Commons Attribution 4.0 International License.

Submitted: December 22, 2020

Accepted: April 1, 2021

Published: May 24, 2021

Reference information: /CI Insight. 2021;6(10):e147134.

https://doi.org/10.1172/jci.

insight.147134.

\section{Introduction}

Percutaneous coronary intervention (PCI) followed by stent implantation currently remains the treatment of choice to treat obstructive coronary arteries. However, the procedure is hampered by in-stent restenosis, a phenomenon characterized by smooth muscle cell (SMC) phenotypic switching, SMC proliferation, and neoatherosclerosis (1). Care of patients with in-stent restenosis remains a prevailing clinical problem. Although drug-eluting stents reduce the incidence of in-stent restenosis, patients are not completely immune to restenosis. Evidence suggests that even with newer generation devices, the restenosis rate is approximately $5 \%-10 \%$ (2). According to the US guidelines, the patients who develop clinical restenosis following drug-eluting stent implantation may again be considered for PCI with balloon angioplasty or drug-eluting stents (3). Therefore, a better understanding of molecular mechanisms that regulate SMC phenotypic switching and proliferation is required to develop effective therapeutic strategies to improve clinical outcomes following PCI.

Integrins are heterodimeric transmembrane receptors responsible for numerous cellular functions, including cell adhesion and migration (4). The integrin family includes $18 \alpha$ and $8 \beta$ subunits that form 24 distinct $\alpha \beta$ heterodimers (5). Unlike other integrins that recognize Arg-Gly-Asp (RGD) sequence, $\alpha 9 \beta 1$ recognizes a Met-Leu-Asp sequence. Genetic knockout studies in mice revealed that phenotypes do not overlap, suggesting different functions in vivo (6). To date, $\beta 1$ is the only reported subunit of $\alpha 9$. Besides SMCs, integrin $\alpha 9 \beta 1$ is expressed on several other cells, including neutrophils, hepatocytes, endothelial cells, and epithelial cells $(7,8)$. The potential mechanistic role of $\alpha 9 \beta 1$ in phenotypic modulation of SMCs and neointimal hyperplasia has not been investigated yet to our knowledge.

Herein, we determined the mechanistic role of integrin $\alpha 9$ in SMC phenotypic modulation in vitro and neointimal hyperplasia in normolipidemic and comorbid conditions of hyperlipidemia. We chose hyperlipidemic conditions in vivo because of the following reasons: first, patients with hyperlipidemia have a higher risk 
of developing restenosis following vascular injury $(9,10)$. PCI procedures are performed in patients who often have elevated levels of cholesterol or triglycerides. Second, hyperlipidemia, a causative factor for neoatherosclerosis, contributes to the progression of in-stent restenosis by potentiating inflammation (11). Herein, utilizing human coronary SMC and SMC-specific integrin $\alpha 9^{-/-}$mice, we demonstrate integrin $\alpha 9 \beta 1$ mechanistically regulates different aspects of SMC biology and promotes neointimal hyperplasia following vascular injury.

\section{Results}

Targeting $\alpha 9$ in stimulated human coronary SMCs suppresses synthetic phenotype and inhibits SMC proliferation and migration. To evaluate the potential role of $\alpha 9 \beta 1$ in SMC biology, we first assessed the time-dependent effect of platelet-derived growth factor-BB (PDGF-BB), which is known to modulate the membrane mobility and trafficking of integrins (12), on $\alpha 9$ expression. Immunoblot analysis suggested an approximately 2-fold increase in integrin $\alpha 9$ expression levels in PDGF-BB-stimulated human coronary SMCs after 6-24 hours $(P<0.05$, Figure 1A). These results were confirmed in parallel by immunofluorescence $(P<0.05$, Figure $1 \mathrm{~B})$. Next, we determined the effect of human-specific anti-integrin $\alpha 9$ antibody (clone Y9A2) on SMC proliferation and migration. Serum-deprived human coronary SMCs were preincubated with Y9A2 $(10 \mu \mathrm{g} / \mathrm{mL})$ for 1 hour and then stimulated with PDGF-BB. SMC morphology was normal during and after treatment. A significant decrease in human coronary SMC proliferation was observed in the group treated with anti-integrin $\alpha 9$ Ig compared with the Ig-treated control (36.11 $\pm 3.4 \%$ vs. $25.8 \pm 2.3 \%$; Figure $1 \mathrm{C})$. In a scratch wound healing assay, we found that anti-integrin $\alpha 9 \mathrm{Ig}$ significantly decreased PDGF-BB-induced human coronary SMC migration (51.9 $\pm 14.2 \%$ vs. $37.2 \pm 1.5 \%$; Figure 1C). Anti-integrin $\alpha 9$ Ig treatment did not exert any significant effect on SMC proliferation or migration in quiescent SMCs. Upon activation, it is known that an SMC undergoes phenotypic modulation from a differentiated "contractile" to a dedifferentiated "synthetic" proliferative phenotype, a process that contributes to proliferation and migration. To determine the role of integrin $\alpha 9$ in SMC phenotypic switching, anti-integrin $\alpha 9$ Ig-treated or Ig-treated human coronary SMCs were stimulated with PDGF-BB and immunostained for contractile (smooth muscle myosin heavy chain 11, SM-MHC; and smooth muscle 22 $\alpha$, SM22 $\alpha$ ) and synthetic (vimentin and osteopontin) phenotype markers (13). We found that the protein levels of contractile markers were higher, whereas synthetic markers were lower in anti-integrin $\alpha 9$ Ig-treated SMCs than Ig-treated cells $(P<0.05$, Figure 1D). Together, these results suggest that blocking integrin $\alpha 9$ signaling with anti-integrin $\alpha 9$ antibody inhibits SMC proliferation and migration and suppresses synthetic phenotype.

Integrin $\alpha 9$ regulates SMC phenotypic switching, proliferation, and migration via focal adhesion kinase/steroid receptor coactivator signaling pathway. Integrins are known to activate signaling pathways, including the activation of focal adhesion kinase (FAK), steroid receptor coactivator (Src), ERK, and p38 MAPK $(4,14)$. We determined whether integrin $\alpha 9$ regulates the FAK/Src pathway, which is known to contribute to cell motility and is a critical signaling event associated with ligand binding to integrins. Quiescent human coronary SMCs pretreated with anti-integrin $\alpha 9$ Ig or control Ig were stimulated with PDGF-BB for 30 minutes. Using immunoblotting, we found that pretreatment with anti-integrin $\alpha 9$ Ig significantly reduced PDGF-BB-induced phosphorylation of FAK ( $\sim 0.6$-fold) and Src ( $\sim 0.6$-fold) when compared with control Ig-treated cells (Figure $2 \mathrm{~A})$. Furthermore, anti-integrin $\alpha 9$ Ig treatment suppressed PDGF-BB-induced activation of ERK1/2 (0.5-fold) and p38 MAPK pathway (0.5-fold) that are linked to cell proliferation and inflammation (Figure 2A). Following integrin engagement, glycogen synthase kinase $3 \beta$ (GSK3 $\beta$ ) phosphorylates $\beta$-catenin, the canonical Wnt pathway's signaling molecule, making it more prone to proteasomal degradation (15). We evaluated whether blocking $\alpha 9$ signaling affects GSK3 $\beta$ and $\beta$-catenin expression. We found that anti-integrin $\alpha 9$ Ig attenuated GSK3 $\beta$ phosphorylation (0.6-fold) that was associated with reduced $\beta$-catenin expression (0.4-fold) when compared with control (Figure $2 \mathrm{~B}$ ). On the other hand, total $\beta$-catenin levels were comparable after $30 \mathrm{~min}$ utes of PDGF-BB stimulation. It is known that $\beta$-catenin translocates to the nucleus after 6 hours of PDGF$\mathrm{BB}$ stimulation (16). We found that nuclear $\beta$-catenin expression, after 6 hours of PDGF-BB stimulation, was significantly reduced in SMCs treated with anti-integrin $\alpha 9$ Ig (0.6-fold) when compared with Ig-treated SMCs (Figure 2B). Anti-integrin $\alpha 9$ Ig treatment did not exert any significant effect on FAK/Src signaling and its downstream targets in quiescent SMCs. Together these results suggest that integrin $\alpha 9$ mechanistically regulates SMC proliferation, migration, and synthetic phenotype via FAK/Src signaling and its downstream targets, including p-ERK, p38 MAPK, and p-GSK3 $\beta / \beta$-catenin signaling.

$S M C$-derived integrin $\alpha 9$ mediates neointimal hyperplasia in mice. We first evaluated the time-dependent effect of PDGF-BB on $\alpha 9$ expression in murine aortic SMCs. Similar to human coronary SMCs, PDGF-BB 
A

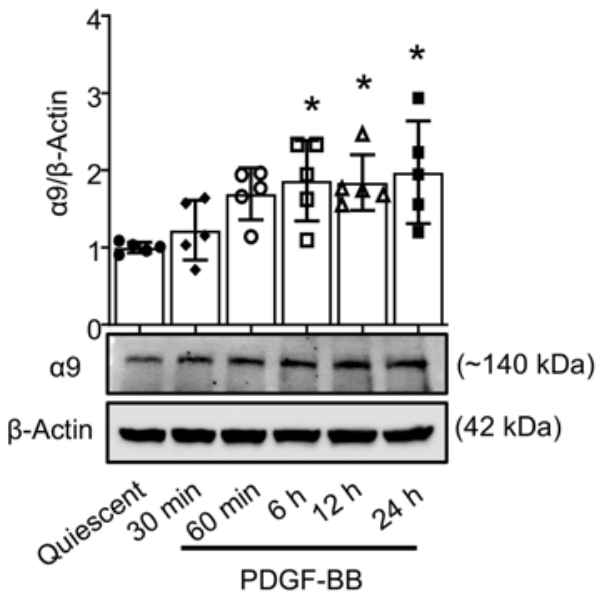

B a9/a-SMA/Hoechst
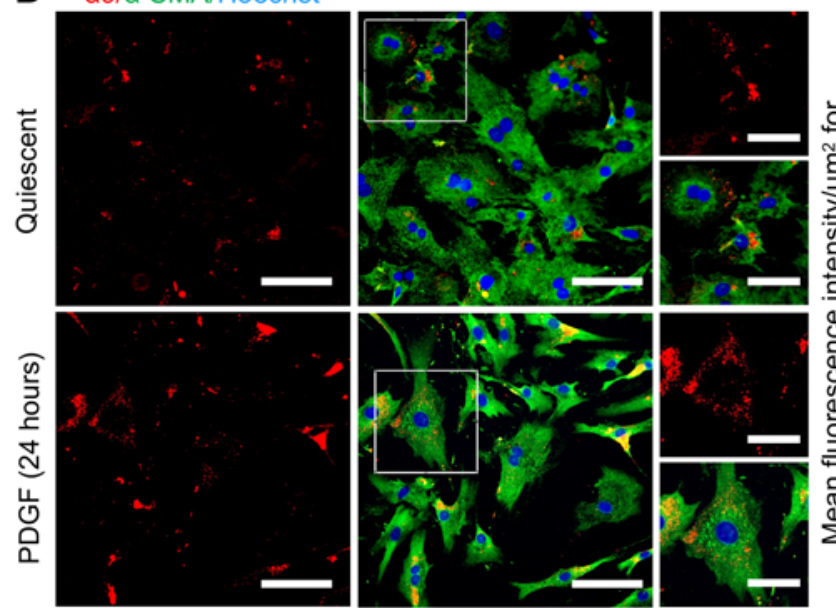

Quiescent $\square$ PDGF-BB

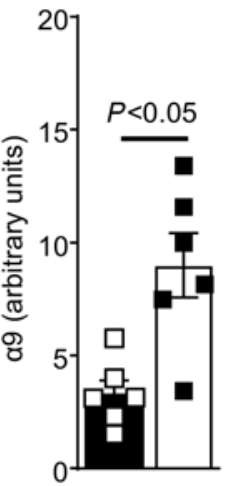

\section{BrdU/a-SMA/Hoechst}
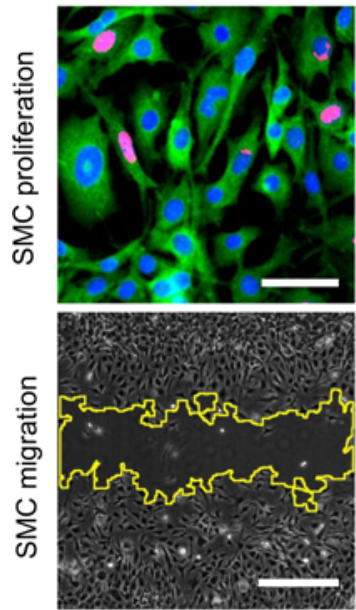

Control-Ig
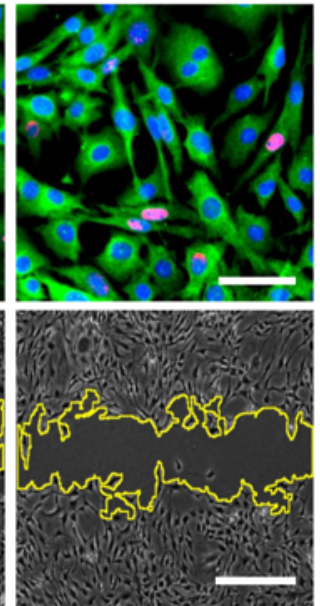

Anti a9-Ig

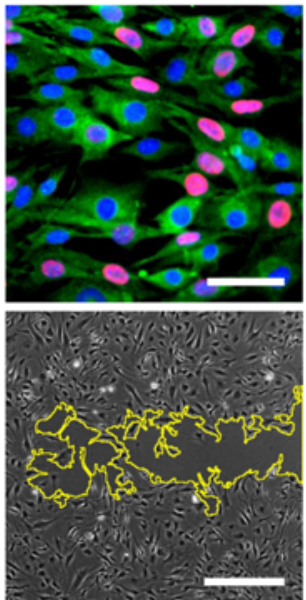

Control-Ig
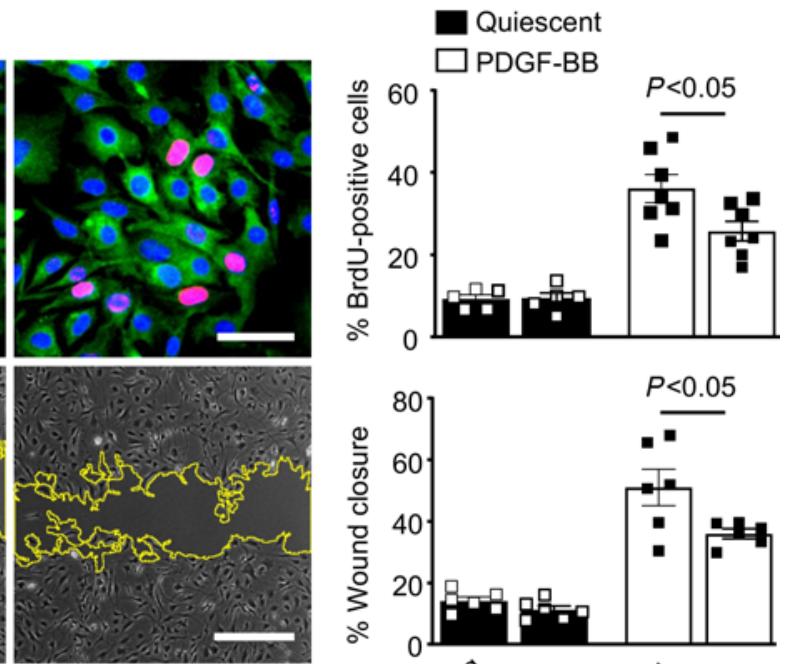

Anti a9-lg

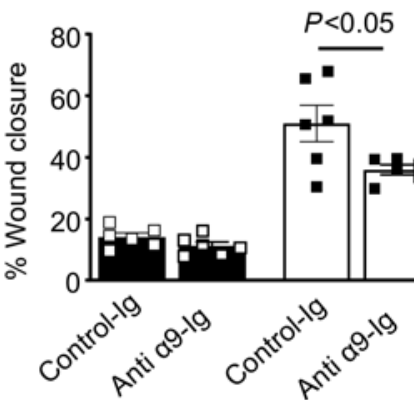

PDGF-BB

\author{
Control-Ig
}

Anti a9-Ig

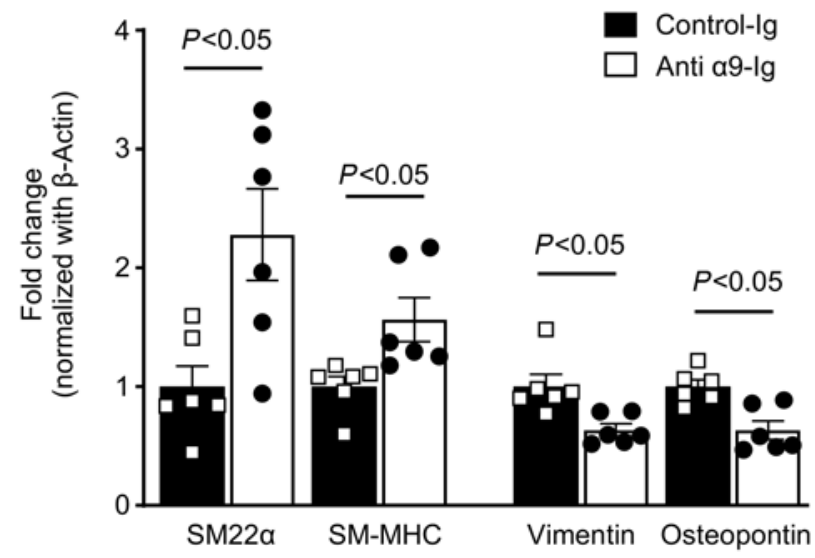

Figure 1. Integrin $\alpha 9$ is upregulated in stimulated human coronary SMCs, and treatment with anti-integrin $\alpha 9$ antibody suppresses synthetic phenotype and inhibits proliferation and migration. (A) Serum-starved human coronary SMCs were stimulated with or without PDCF-BB for indicated time points. Representative immunoblots and densitometric analysis of $\alpha 9$ and expression levels. $\beta$-Actin was used as a loading control ( $n=5 / \mathrm{group})$. (B) The left panels show representative double immunostaining for $\alpha 9$ (shown in red) and SMC actin ( $\alpha$ SMA) (shown in green) in SMCs stimulated with or without PDCF-BB for 24 hours. Boxed regions are magnified (scale bars: $25 \mu \mathrm{m}$ ). Scale bars: $50 \mu \mathrm{m}$. The right panel shows the quantification of $\alpha 9$ fluorescence intensity $(n=6 / g r o u p)$. 
(C and D) Quiescent human coronary SMCs were pretreated with anti- $\alpha 9$ blocking antibody (clone Y9A2, $10 \mu \mathrm{g} / \mathrm{mL}$ ) for 60 minutes. (C) Human coronary SMCs were stimulated with PDCF-BB for 24 hours. The top panels show representative BrdU-positive cells (red) costained with $\alpha$ SMA (green) and Hoechst (blue). Scale bars: $50 \mu \mathrm{m}$. The bottom panels show representative phase-contrast images of SMC migration in the scratch assay. Scale bars: $500 \mu \mathrm{m}$. The right panel shows the quantification of BrdU-positive cells to the total number of cells ( $n=6 /$ group) and migrated area ( $n=6 /$ group). (D) Representative immunoblots and densitometric analysis of SM22 $\alpha$, SM-MHC, vimentin, and osteopontin ( $n=6 /$ group) in human coronary SMCs stimulated with PDCF-BB for 24 hours. \#1 and \#2 represent 2 separate samples. Statistical analysis: (A) 1-way ANOVA with Bonferroni's post hoc test; (C) 2-way ANOVA followed by uncorrected Fisher's least significant differences (LSD) test (B and D) unpaired 2-tailed Student's $t$ test. ${ }^{*} P<0.05$ vs. quiescent or vehicle-treated (control lg) groups.

stimulation led to a time-dependent increase in $\alpha 9$ expression in murine aortic SMCs $(P<0.05$, Figure $3 \mathrm{~A})$. In line with these results, $\alpha 9$ mRNA expression levels were increased following PDGF-BB stimulation compared with control quiescent SMCs $(P<0.05$, Figure 3B). In parallel, we found that $\alpha 9$ expression was higher in the injured carotid artery at 28 days when compared with the uninjured control artery (Supplemental Figure 1; supplemental material available online with this article; https://doi.org/10.1172/jci. insight.147134DS1). $\beta 1$ is the only reported subunit of $\alpha 9$; therefore, genetic deletion of $\alpha 9$ should inhibit $\alpha 9 \beta 1$-mediated signaling only. To show a definitive role for $\alpha 9 \beta 1$ in SMC biology and neointimal hyperplasia in the comorbid condition of hyperlipidemia, we generated SMC-specific $\alpha 9$-deficient mice on apolipoprotein E-deficient $\left(\mathrm{Apoe}^{-/}\right)$background $\left(\alpha 9^{\mathrm{A} / \mathrm{l}} \mathrm{SM} 22 \alpha \mathrm{Cre}^{+} \mathrm{Apoe}^{-/-}\right.$; hereafter for simplicity referred to as $\alpha 9^{\text {SMC-Ko; }}$ Supplemental Figure 2A). The SM22 $\alpha \mathrm{Cre}^{+}$transgenic mice expressed Cre recombinase under the control of mouse SMC protein $22 \alpha$ (or transgelin) promoter. Genomic PCR confirmed the presence of the SM22 $\alpha$ Cre gene in $\alpha 9^{\mathrm{f} / \mathrm{ll}} \mathrm{Apoe}^{-/-}$mice (Supplemental Figure 2B). Previously, it was shown that the global deletion of $\alpha 9$ in mice results in a defect in granulopoiesis (17). In contrast, we found that complete blood counts were comparable between $\alpha 9^{\text {SMc-Ko }}$ and control $\alpha 9^{9^{1 / 1 / 1}}$ mice (Supplemental Table 1), suggesting that SMC-specific deletion of $\alpha 9$ does not result in impaired granulopoiesis and that $\alpha 9^{\text {SMC-KO }}$ could be used as an appropriate model to evaluate the role of integrin $\alpha 9$ in SMC biology. Western blot analysis confirmed the lack of integrin $\alpha 9$ expression in SMCs isolated from the $\alpha 9^{\text {SMC-KO }}$ mice (Figure 3C). Genetic deletion of integrin $\alpha 9$ in SMCs did not affect $\beta 1$ subunit expression (Supplemental Figure 3). Susceptibility to neointimal hyperplasia was studied at 28 days following wire injury in the carotid artery of littermate $\alpha 9^{\mathrm{n} / \mathrm{fl}}$ and $\alpha 9^{\text {SMC-KO }}$ mice. Male and female mice were used to determine sex-based differences. SMC-specific integrin $\alpha 9$ deletion led to a significant decrease in neointimal area and neointima/media ratio $\left(P<0.05\right.$ versus $\alpha 9^{\mathrm{n} / \mathrm{fl}}$; Figure 3D). The medial area was comparable between $\alpha 9^{\mathrm{fl} / \mathrm{ll}}$ and $\alpha 9^{\text {SMC-KO }}$ mice (Figure 3D). Immunostaining revealed that $\alpha 9$ expression was minimal and mainly restricted to the luminal area in $\alpha 9^{\text {sмс-ко mice }}$ (Supplemental Figure 4). Next, we analyzed SMC proliferation and apoptosis in wire-injured carotid artery sections because of the differential role in neointima formation. We found that SMC proliferation was significantly reduced in $\alpha 9^{\text {SMC-Ko }}$ mice when compared with control $\alpha 9^{\text {fi/li }}$ mice (Figure $3 \mathrm{E}$ ). Next, we analyzed BrdU incorporation in the neointima and the media. We found that percentage of BrdU-positive cells was

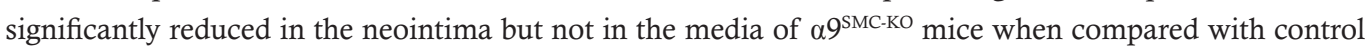
$\alpha 9^{\mathrm{A} / \mathrm{l}}$ mice (Supplemental Figure 5). SMC-specific $\alpha 9$ deletion did not affect apoptosis (Figure 3F). Together these results suggest a mechanistic role for SMC-derived integrin $\alpha 9$ in vascular remodeling.

$S M C$-specific integrin $\alpha 9$ regulates SMC phenotypic switching, proliferation, and migration via FAK/Src, ERK, p38, and GSK3 $\beta / \beta$-catenin pathway. Cultured SMCs from $\alpha 9^{\mathrm{A} / \mathrm{l}}$ and $\alpha 9^{\text {sMC-Ko }}$ mice were subjected to BrdU incorporation and scratch wound assays to obtain further mechanistic insights. We found that SMC-specific $\alpha 9$ deletion led to a significant decrease in PDGF-BB-induced SMC proliferation $(49.8 \% \pm 2.8 \%$ vs. $33.5 \% \pm 1.0 \%$; Figure $4 \mathrm{~A}$ ) and SMC migration ( $50.8 \% \pm 3.5 \%$ vs. $33.5 \% \pm 3.1 \%$; Figure $4 \mathrm{~A})$ when compared with control. To elucidate the role of integrin $\alpha 9$ on SMC phenotypic switching, PDGF-BB-stimulated SMCs from $\alpha 9^{\text {f1/ }}$ and $\alpha 9^{\text {SMC-Ko }}$ mice were subjected to Western blotting assay. We found that the expression levels of contractile markers (SM22 $\alpha$ and SM-MHC) were higher than control. In contrast, synthetic markers (vimentin and osteopontin) were lower in SMCs isolated from $\alpha 9^{\text {SMC-KO }}$ mice when compared with SMCs from $\alpha 9^{\text {fl/l }}$ mice (Figure 4B). These results were confirmed in parallel by immunofluorescence (Figure 4C). Together, these results suggest that genetic ablation of integrin $\alpha 9$ prevents SMC proliferation, migration, and phenotypic switching.

We next determined whether SMC-specific deletion of integrin $\alpha 9$ limits PDGF-BB-induced activation of FAK, Src, ERK, p38, and GSK3 $3 / \beta$-catenin signaling, similar to that observed in human coronary SMCs treated with anti-integrin $\alpha 9 \mathrm{Ig}$. Using immunoblotting, we found that after 30 minutes of PDGF-BB stimulation,

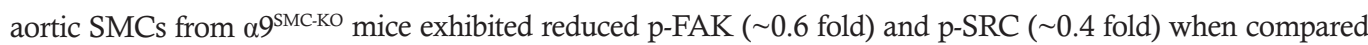
with control $\alpha 9^{\text {f/ll }}$ mice (Figure 5A). Furthermore, deletion of $\alpha 9$ suppressed PDGF-BB-induced p-ERK1/2 (0.5-fold) and p-p38 expression (0.4-fold) (Figure 5A). We also found that deletion of $\alpha 9$ attenuated GSK3 $\beta$ 
A

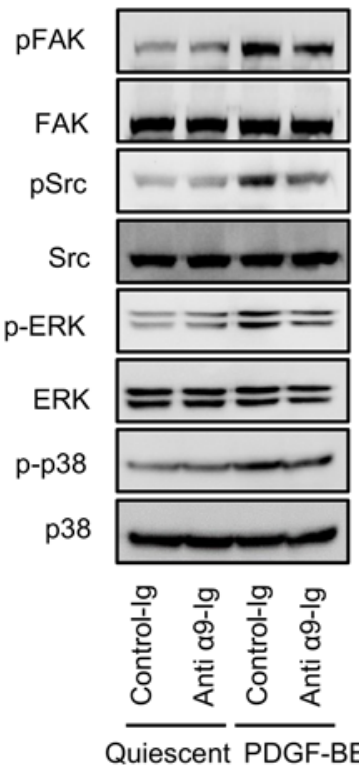

(125 kDa)

$\square$ Quiescent + Control-Ig

(40 kDa)

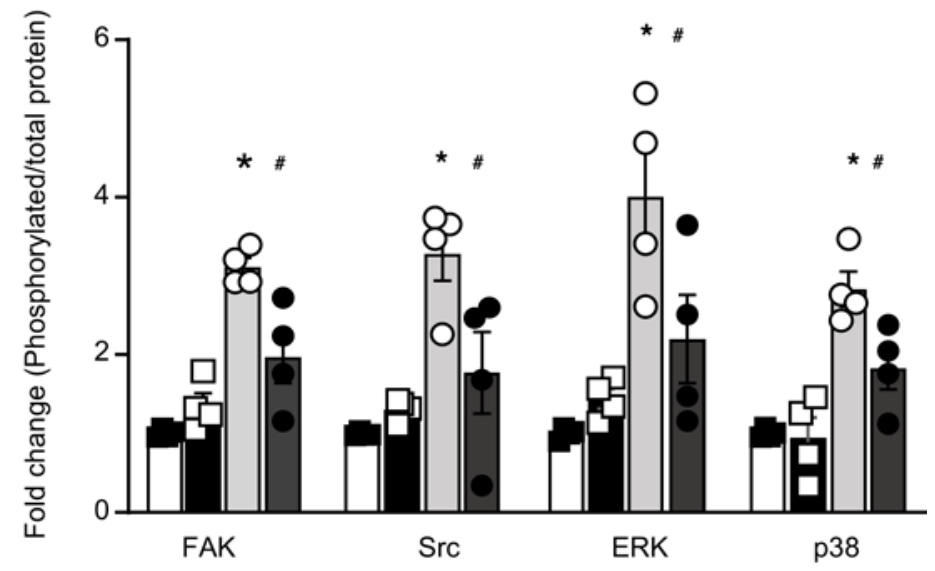

B Whole Cell Lysate

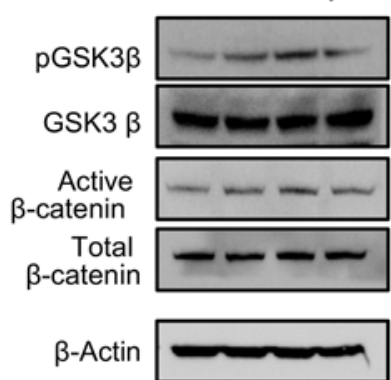

(46 kDa)

(46 kDa)

(92 kDa)

(92 kDa)

$(42 \mathrm{kDa})$

Nuclear extract

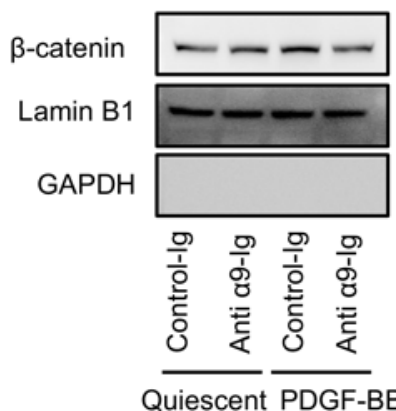

$\square$ Quiescent + Control-Ig

Quiescent + Anti a9-Ig

PDGF-BB + Control-Ig

PDGF-BB + Anti a9-Ig

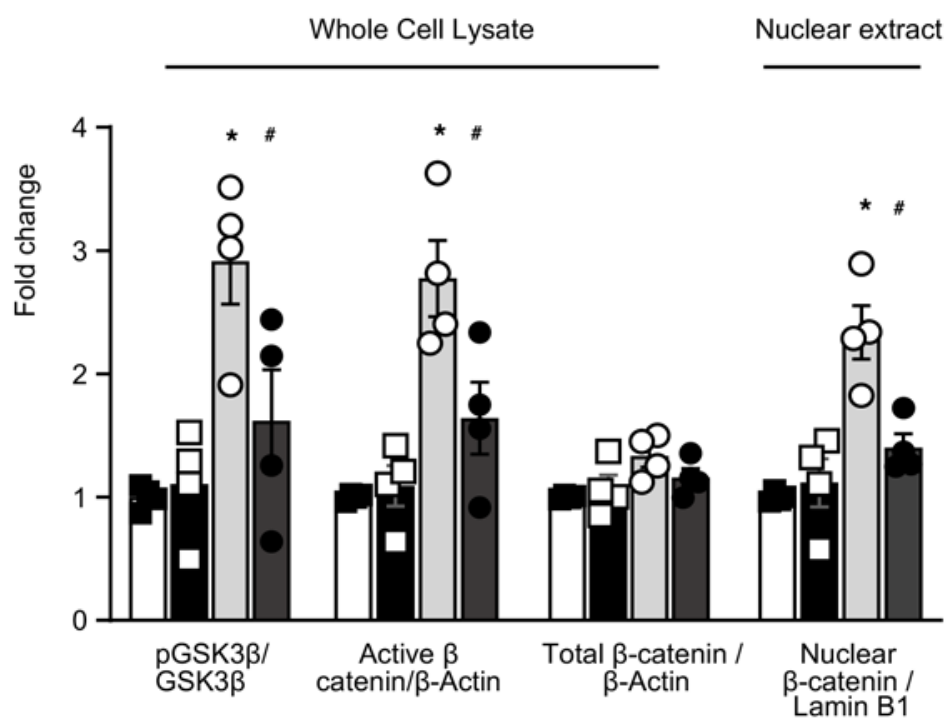

Figure 2. Anti-integrin $\alpha 9$ antibody suppresses FAK/Src, ERK, p38, and glycogen synthase kinase $\mathbf{3 \beta} / \boldsymbol{\beta}$-catenin pathway. Serum-starved human coronary SMCs were stimulated with or without PDGF-BB. (A) Representative Western blots and densitometric analysis of FAK, Src, ERK1/2, p38, and $\beta$-actin after 30 minutes of PDGF-BB stimulation ( $n=4 /$ group). (B) Representative Western blots and densitometric analysis of GSK3 $\beta$, $\beta$-catenin, and $\beta$-actin after 30 minutes of PDGF-BB stimulation. Nuclear extracts were prepared after 6 hours of PDGF-BB stimulation. $\beta$-Catenin and Lamin B1 were detected by immunoblotting ( $n=4$ /group). Statistical analysis: 2 -way ANOVA followed by uncorrected Fisher's LSD test. ${ }^{*} P<0.05$ vs. quiescent + control lg; ${ }^{\#} P<0.05$ vs. PDGF-BB + control lg-treated groups. p-ERK, phosphorylated ERK.

phosphorylation (0.6-fold). Concomitantly a decrease in nonphosphorylated $\beta$-catenin expression (0.5-fold) was noted in $\alpha 9$-deficient SMCs compared with control (Figure $5 \mathrm{~B}$ ). No significant change in total $\beta$-catenin expression was observed in $\alpha 9$-deficient SMCs when compared to control. However, nuclear $\beta$-catenin expression was significantly reduced in SMCs from $\alpha 9^{\text {SMC-KO }}$ mice compared with control (0.6-fold, Figure $\left.5 \mathrm{~B}\right)$. We further explored how $\alpha 9$ deletion downregulates $\beta$-catenin expression. SMCs were stimulated with PDGF-BB in the 
$\mathbf{A}$
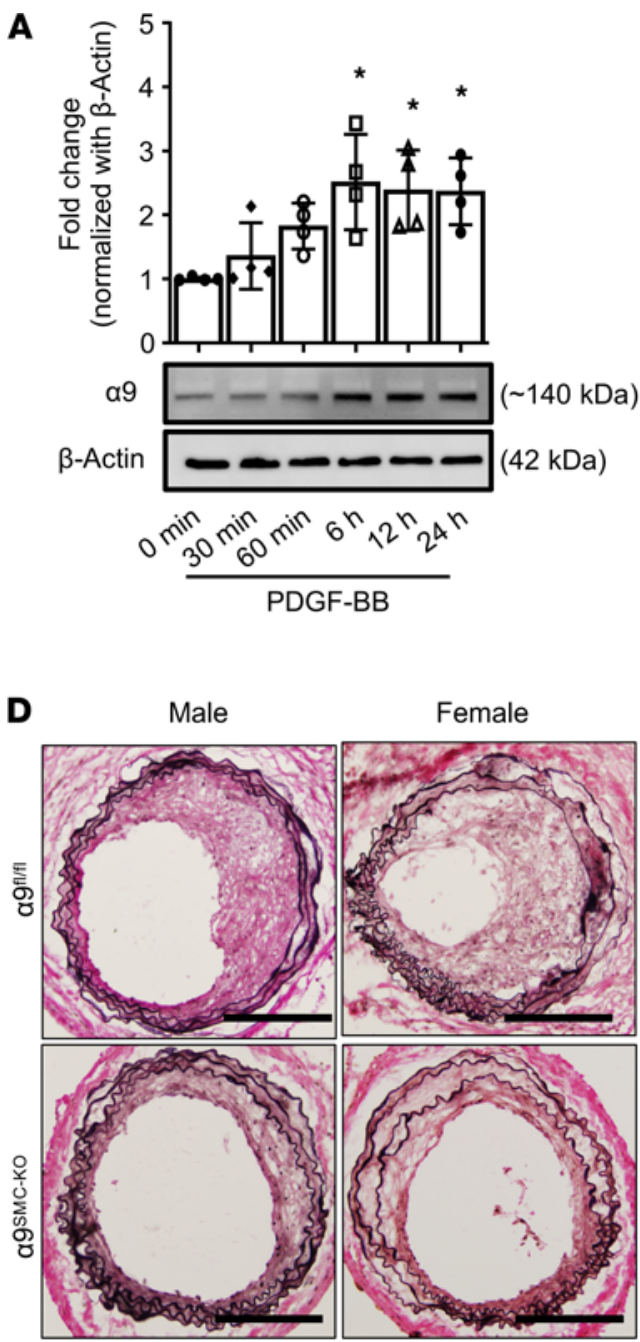

Day 28

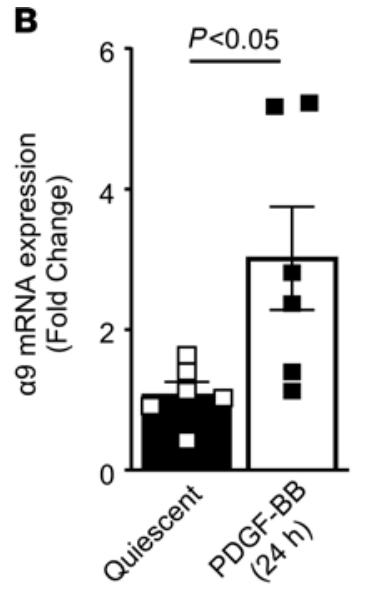

C

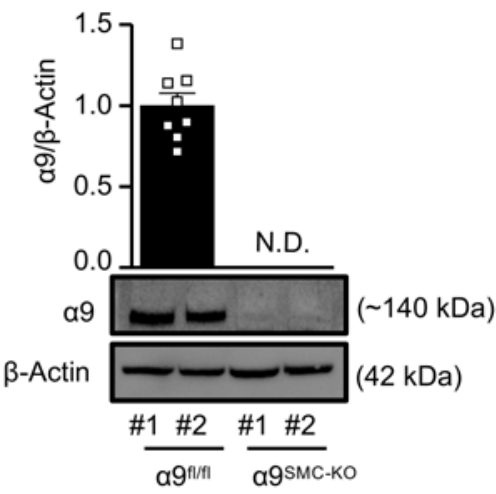

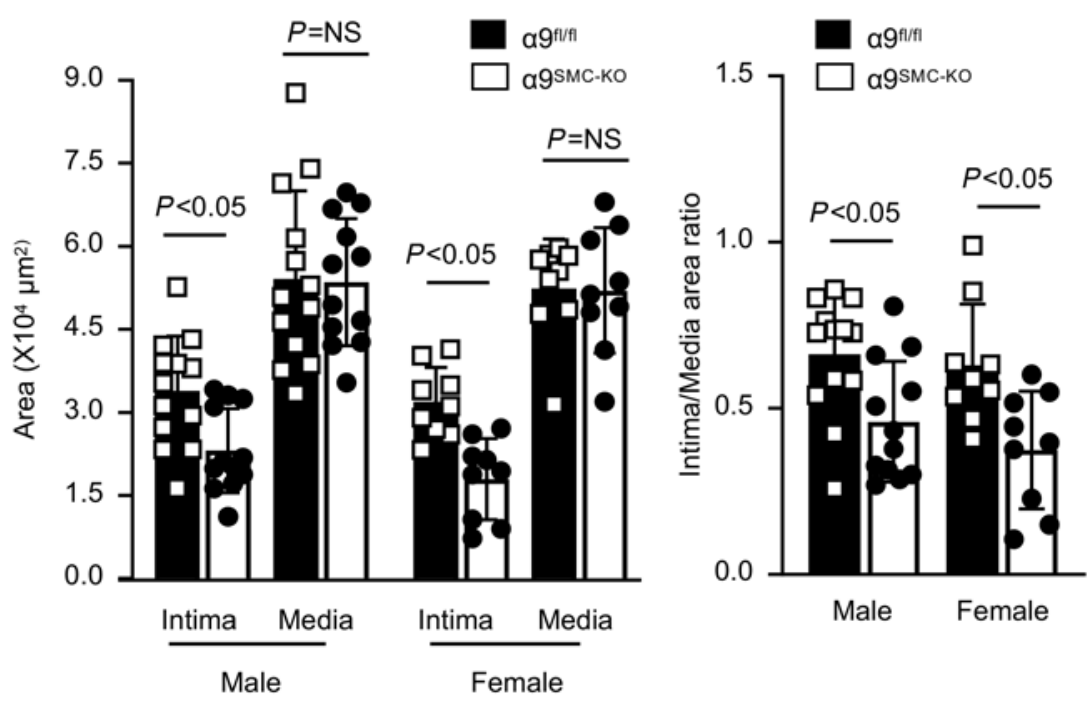

E BrdU/aSMA/Hoechst
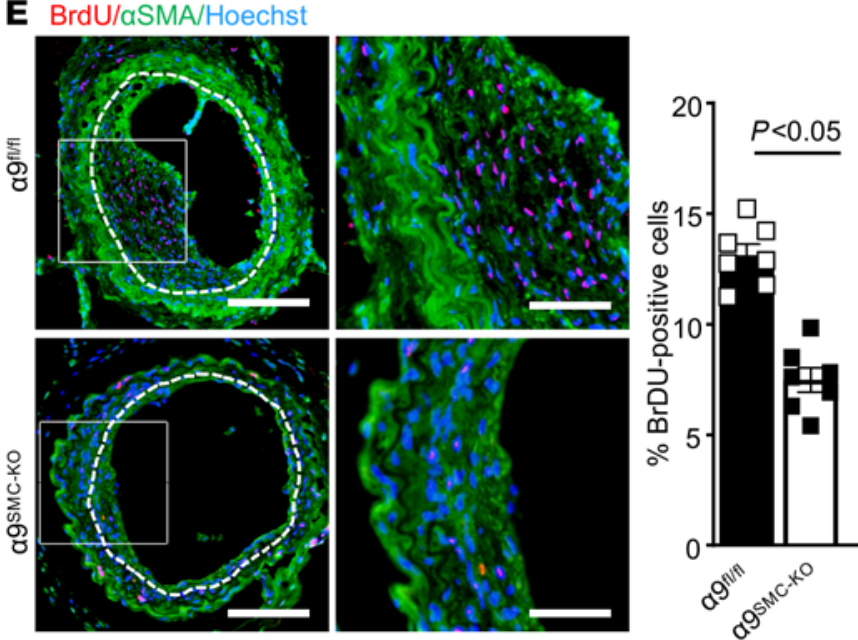

F TUNEL/Hoechst
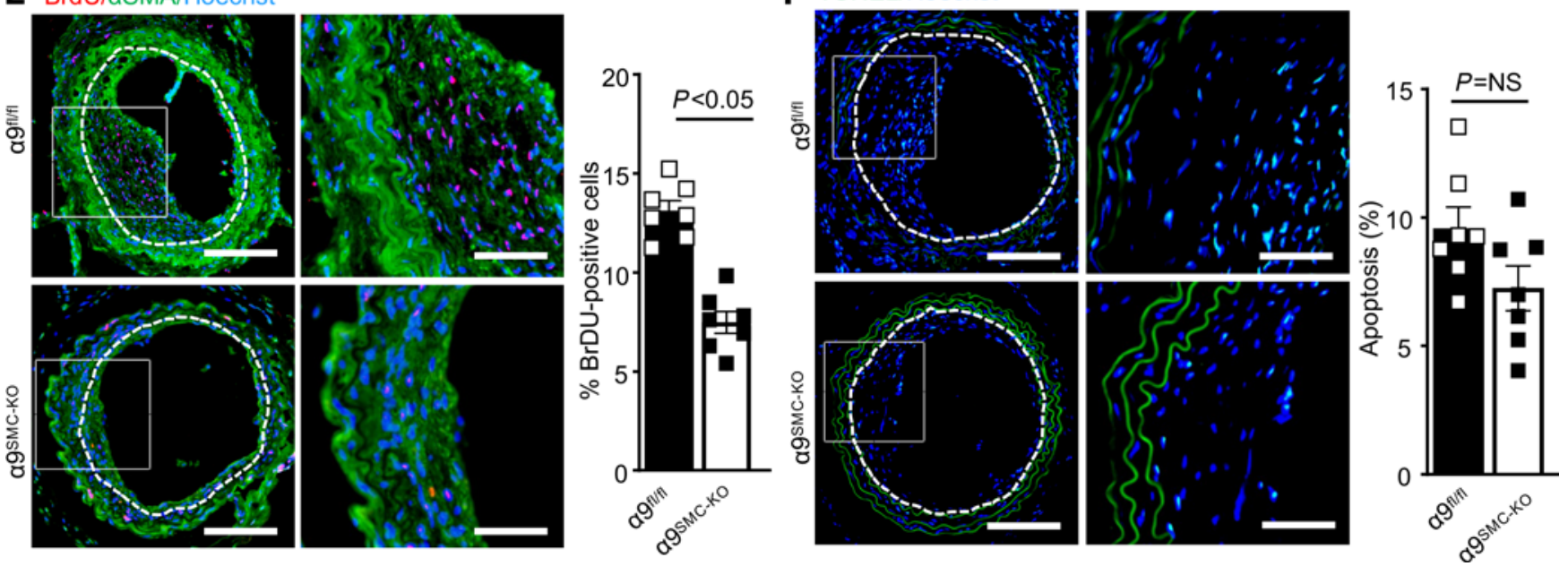

Figure 3. SMC-specific integrin $\alpha \mathbf{9}$ modulates neointimal hyperplasia in mice. All mice are on $\mathrm{Apoe}^{-/-}$background. Serum-deprived SMCs were stimulated with or without PDGF-BB $(20 \mathrm{ng} / \mathrm{mL})$ for indicated time points. (A) Representative immunoblots and densitometric analysis of $\alpha 9$ expression levels. $\beta$-Actin was used as a loading control $(n=4)$. (B) Quantification of $\alpha 9$ mRNA expression by real-time PCR $(n=6)$. (C) Western blot analysis of $\alpha 9$ and $\beta$-actin in SMCs isolated from $\alpha 9^{\text {SMC-KO }}$ and control $\alpha 9^{f / / f l}$ mice. \#1 and \#2 represent samples from 2 individual mice. (D) The left panels show representative photomicrographs of Verhoeff's van Gieson-stained carotid artery sections from male and female $\alpha 9^{5 \mathrm{Mc}-\mathrm{ko}}$ and control $\alpha$ 9 $^{\mathrm{fl} / \mathrm{fl}}$ mice after 28 days of injury (male $n=12-13$; female $n=9$ /group). Scale bars: $200 \mu \mathrm{m}$. The right panels show quantification of intimal area, medial area, and a ratio of intimal to medial 
area. Each dot represents a single mouse. (E) The left panels show representative BrdU-positive cells (red) counterstained with $\alpha$ SMA (green). Nuclei are counterstained with Hoechst (blue). The right panel shows the quantification of percentage BrdU-positive cells ( $n=7$ ). Scale bars: $200 \mu m$. (F) The left panels show representative TUNEL-positive cells (green) counterstained with Hoechst (blue). The right panel shows the quantification of TUNEL-positive cells $(n=7)$. Scale bars: $200 \mu \mathrm{m}$. Values are represented as mean \pm SEM. Statistical analysis: (A) 1-way ANOVA followed by Bonferroni's post hoc test; (B-F) unpaired 2-tailed Student's $t$ test. ${ }^{*} P<0.05$ versus quiescent. N.D., not detected.

presence or absence of proteasome-specific inhibitor (PSI, $10 \mu \mathrm{M})$. Expression of $\beta$-catenin protein was monitored in the nuclear extract after 6 hours of PDGF-BB stimulation. We found that PSI treatment significantly recovered $\beta$-catenin protein level in $\alpha$-deficient SMCs, suggesting that integrin $\alpha 9$ promotes $\beta$-catenin degradation through proteasome pathway (Supplemental Figure 6). Several genes are regulated by the Wnt/ $\beta$-catenin pathway, including matrix metalloproteinase 2 (MMP-2), MMP-9 (18), cyclin D1 (19), and c-Myc (20), which are known to contribute to SMC migration and proliferation. We found that SMC-specific integrin $\alpha 9$ deletion reduced PDGF-BB-induced transcription of MMP-2, MMP-9, cyclin D1, and c-Myc (Figure 5C).

Extracellular matrix protein fibronectin containing extra domain A partially contributes to integrin $\alpha 9-$ mediated SMC proliferation, migration, and phenotypic switching. The extracellular matrix protein fibronectin containing extra domain A (Fn-EDA) is an endogenous ligand for integrin $\alpha 9 \beta 1$ (21). To determine whether SMC-specific integrin $\alpha 9$ mediates SMC proliferation and migration via Fn-EDA, we treated SMCs from $\alpha 9^{\mathrm{fl} / \mathrm{fl}}$ and $\alpha 9^{\mathrm{SMC}-\mathrm{KO}}$ mice with recombinant EDA-containing [EDA (+)] or EDA-lacking [EDA (-)] peptides $(10 \mu \mathrm{g} / \mathrm{mL})$. We found that EDA (+) peptide significantly increased proliferation and migration of SMCs isolated from $\alpha 9^{\text {ft/fl }}$ mice (Figure $6 \mathrm{~A})$. Furthermore, EDA (+) peptide increased the expression levels of synthetic markers (vimentin and osteopontin) and decreased the expression of contractile markers (SM22 $\alpha$ and SM-MHC) in SMCs of $\alpha 9^{\text {fl/fl }}$ mice (Figure 6B). EDA (+) peptide also increased proliferation and migration of SMCs of $\alpha 9^{\text {SMC-KO }}$ mice, suggesting a role of other EDA ligands in addition to $\alpha 9 \beta 1$ in SMC proliferation and migration. It is important to note

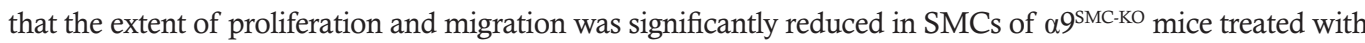
EDA (+) peptide compared with SMCs of $\alpha 9^{\text {fl/fl }}$ mice treated with EDA (+) peptide (Figure 6A), suggesting Fn-EDA partially contributes to integrin $\alpha 9-$ mediated SMC proliferation and migration. Similarly, EDA (+) peptide increased the expression of synthetic markers (vimentin and osteopontin) and decreased the expression

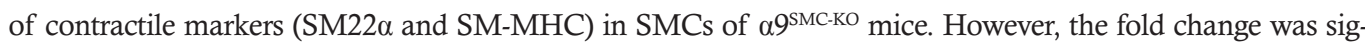
nificantly less than the SMCs of $\alpha 9^{\mathrm{f} / \mathrm{fl}}$ mice treated with EDA (+) peptide (Figure 6B). Previous reports suggest that fibronectin-EDA interaction with integrin $\alpha 9$ results in activation of FAK, ERK, p38, and $\beta$-catenin signaling pathways (22). We determined activation of these proteins in $\alpha 9$-deficient SMCs treated with recombinant EDA-containing or -lacking peptide. Using immunoblotting, we found that EDA (+) peptide significantly increased the expression of p-FAK, p-ERK1/2, and p-p38 in SMCs from control $\alpha 9^{\mathrm{A} / \mathrm{fl}}$ mice (Figure 6C). In line with these observations, EDA $(+)$ peptide treatment significantly increased nuclear $\beta$-catenin expression in SMCs from $\alpha 9^{\mathrm{f} / \mathrm{fl}}$ mice. On the other hand, genetic deletion of $\alpha 9$ partially inhibited EDA $(+)$ peptide-induced increased expression of p-FAK, p-ERK1/2, p-p38, and nuclear $\beta$-catenin expression (Figure 6C). Together these results suggest that Fn-EDA partially contributes to $\alpha 9$-mediated SMC proliferation and migration.

Targeting integrin $\alpha 9 \beta 1$ signaling using a specific inhibitor reduces neointimal hyperplasia in wild-type mice by limiting SMC proliferation and migration. We next evaluated the therapeutic potential of targeting integrin $\alpha 9$ to reduce vascular injury-induced neointimal hyperplasia. Serum-deprived SMCs from wild-type (WT, C57BL/6J) mice were preincubated with murine anti-integrin $\alpha 9$ antibody (clone 55A2C, $10 \mu \mathrm{g} / \mathrm{mL}$, refs. 23-25) for 1 hour and then stimulated with PDGF-BB. SMC morphology was normal during and after treatment. We found that SMC proliferation and migration were significantly attenuated in the group treated with anti-integrin $\alpha 9$ Ig when compared with the control Ig-treated group (Figure 7A). To determine any off-target effects of $55 \mathrm{~A} 2 \mathrm{C}(10 \mu \mathrm{g} / \mathrm{mL})$, aortic SMCs derived from $\alpha 9^{\text {SMC-КO }}$ mice were treated with $55 \mathrm{~A} 2 \mathrm{C}$ for 60 minutes prior to PDGF-BB stimulation, and SMC proliferation and migration were measured after 24 hours. No inhibitory effect of 55A2C was observed on SMC proliferation or migration in SMCs lacking $\alpha 9$ (Supplemental Figure 7), suggesting that 55A2C at a dose of $10 \mu \mathrm{g} / \mathrm{mL}$ does not exert any off-target effect. We also analyzed mRNA expression of genes regulated by $\alpha 9$ in SMCs from WT mice pretreated with $\alpha 9$ blocking antibody. We found that treatment with anti-integrin $\alpha 9$ Ig significantly inhibited PDGFBB-induced upregulation of $\beta$-catenin-regulated genes including MMP-2, MMP-9, c-Myc, and cyclin D1 (Supplemental Figure 8). Male WT mice were randomly assigned to receive either anti-integrin $\alpha 9 \operatorname{Ig}(200$ $\mu \mathrm{g} /$ mouse, intravenous bolus injection) or control Ig, 60 minutes prior to vascular (wire) injury. Susceptibility to neointimal hyperplasia was evaluated 4 weeks after vascular injury. Anti-integrin $\alpha 9$ Ig-treated mice 
A BrdU/a-SMA/Hoechst

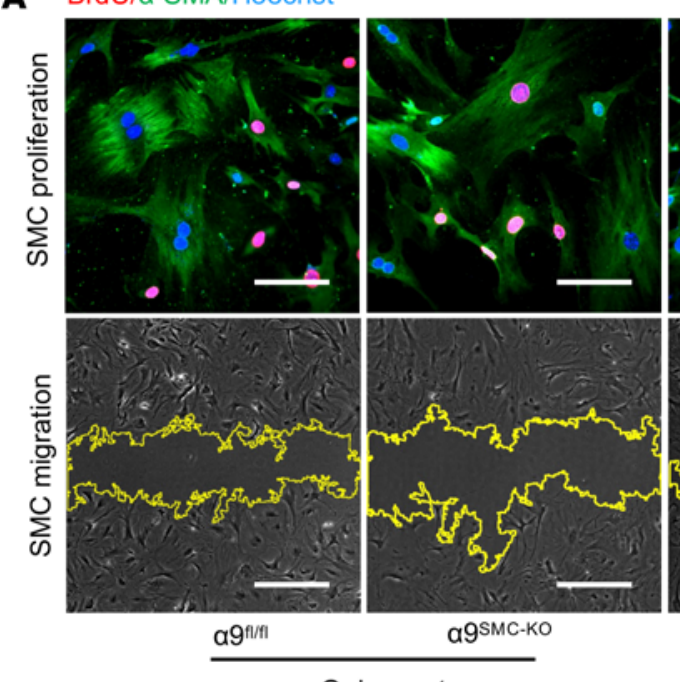

Quiescent

B
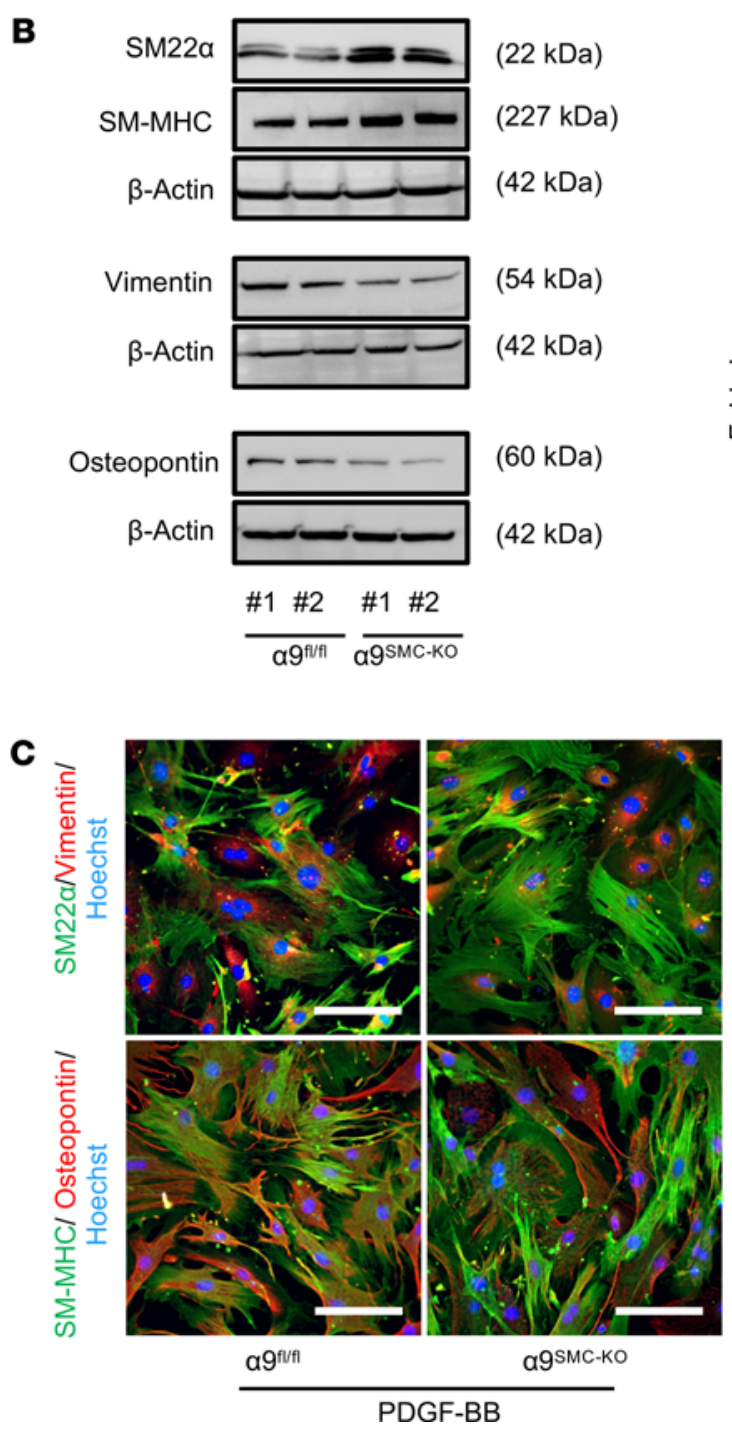
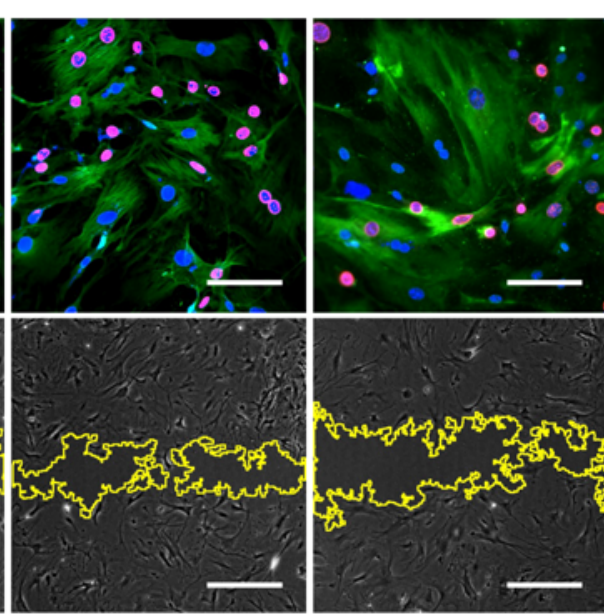

a9f//1

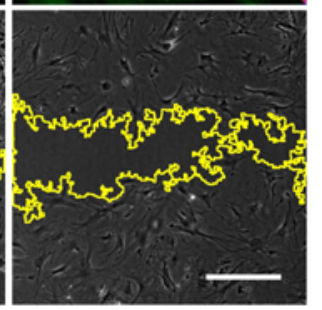

a9smc-ko
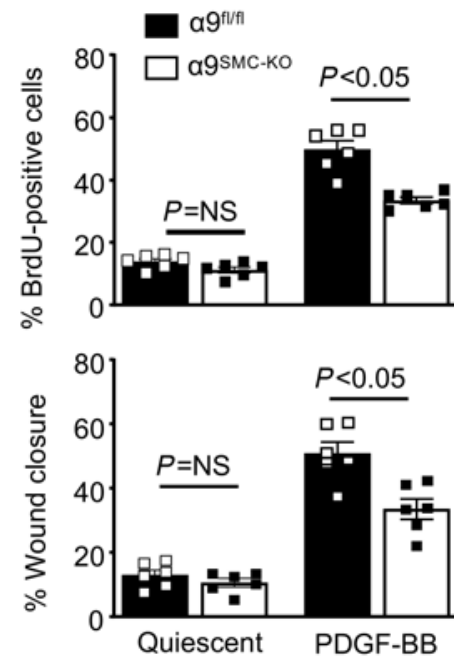

PDGF-BB
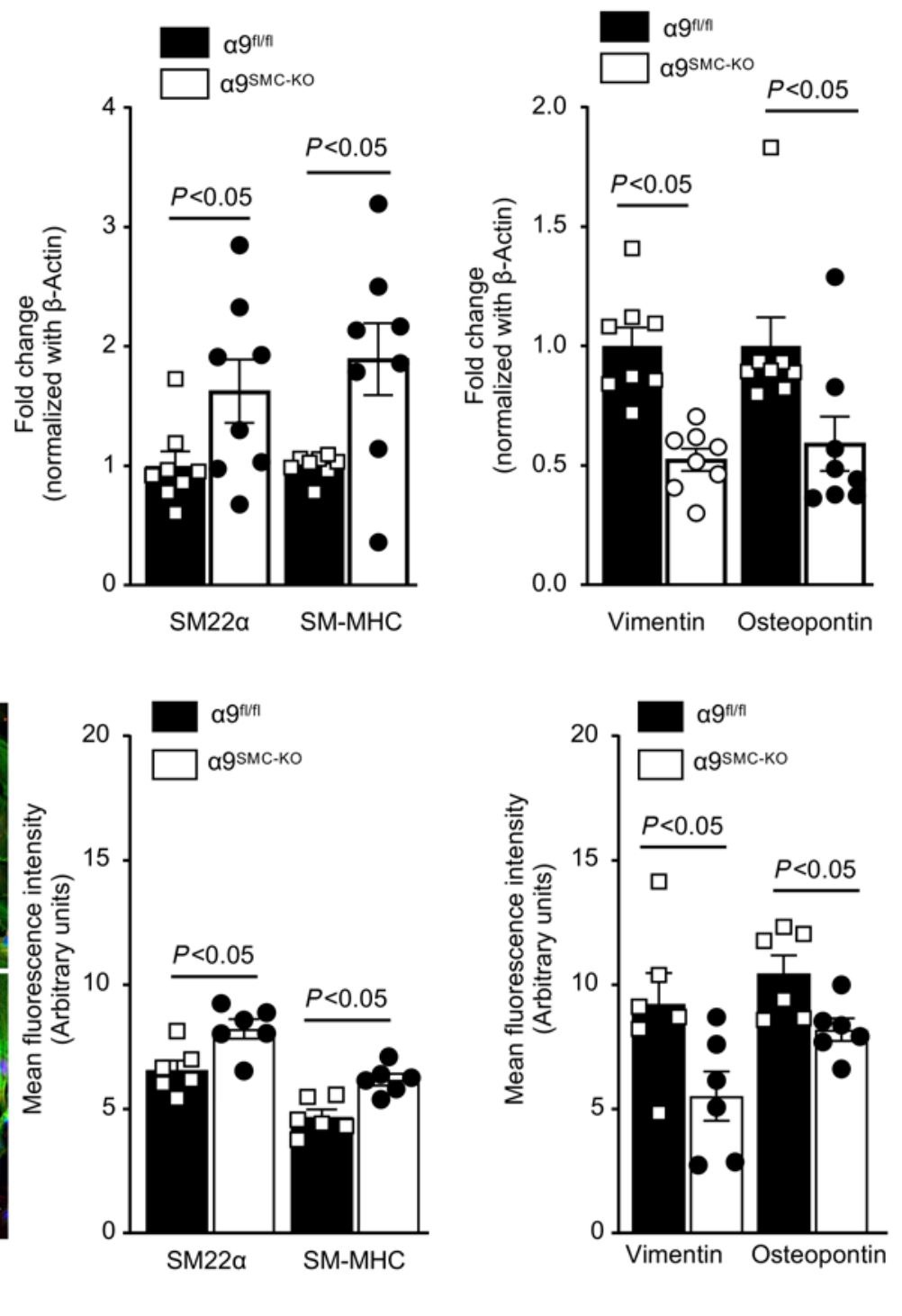
Figure 4. SMC-specific $\alpha 9$ deletion suppresses PDGF-BB-induced SMC proliferation, migration, and phenotypic switching. Aortic SMCs isolated from $\alpha 9^{\mathrm{SMC}-\mathrm{KO}}$ and control $\alpha 9^{\mathrm{fl} / \mathrm{fl}}$ mice were serum-starved and stimulated with PDGF-BB for 24 hours. (A) The left upper panels show representative BrdU-positive cells (red) costained with $\alpha$ SMA (green) and Hoechst (blue). Scale bars: $50 \mu \mathrm{m}$. The left lower panels show representative phase-contrast images of SMC migration in the scratch assay. Scale bars: $500 \mu \mathrm{m}$. The right panel shows the quantification of BrdU-positive cells to the total number of cells and quantification of the migrated area $(n=6)$. (B) Representative immunoblots and densitometric analysis of contractile proteins (SM22 $\alpha$ and SM-MHC) and synthetic proteins (vimentin and osteopontin) $(n=8)$. (C) The left panels show representative immunostained images for contractile proteins (SM22 $\alpha$, green; and $\mathrm{SM}-\mathrm{MHC}$, green) and synthetic proteins (vimentin, red; and osteopontin, red). Scale bars: $50 \mu \mathrm{m}$. The right panels show quantification of the fluorescence intensity for SM22 $\alpha$, SM-MHC, vimentin, and osteopontin ( $n=6$ per group). Values are expressed as mean \pm SEM. Statistical analysis: (A) 2-way ANOVA followed by uncorrected Fisher's LSD test; (B and C) unpaired 2-tailed Student's $t$ test.

exhibited a marked decrease in the neointimal area and neointimal/medial ratio $(P<0.05$ vs. vehicle-treated control mice; Figure 7B). Next, we analyzed SMC proliferation and apoptosis in wire-injured carotid artery sections. We found that SMC proliferation was significantly reduced in mice treated with anti-integrin $\alpha 9$ antibody as compared with control Ig-treated mice (Figure 7C). Further analysis revealed that the percentage of BrdU-positive cells was significantly reduced in the neointima but not in the media of $\alpha 9$ antibodytreated mice as compared with control Ig-treated mice (Supplemental Figure 9). Immunostaining revealed an increase in contractile proteins (SM22 $\alpha$ and SM-MHC) and a decrease in synthetic proteins (vimentin and osteopontin) in the neointima of anti-integrin $\alpha 9 \mathrm{Ig}$-treated mice as compared with control Ig-treated mice (Supplemental Figure 10). Anti-integrin $\alpha 9$ antibody treatment did not affect apoptosis (Figure 7D).

\section{Discussion}

The results of this study suggest that integrin $\alpha 9 \beta 1$ is a key factor that regulates several aspects of SMC biology and vascular remodeling. We believe that these findings are novel and may have clinical significance for the following reasons: first, stimulated human coronary SMCs upregulate integrin $\alpha 9$ expression, which may be a prerequisite to coronary SMC proliferation and neointimal hyperplasia. Second, utilizing anti-integrin $\alpha 9$ antibody or SMC-specific $\alpha 9$-deficient mice, we demonstrate integrin $\alpha 9 \beta 1$ regulates SMC phenotype switching, proliferation, and migration. SMC-specific $\alpha 9$-deficient mice exhibited reduced injury-induced neointimal hyperplasia. Finally, we provide evidence that deletion of SMC-specific integrin $\alpha 9$ was associated with reduced activation of FAK/Src, ERK, p38, and GSK3 $\beta / \beta$-catenin pathway.

Several integrins, including $\alpha 2 \beta 1, \alpha 5 \beta 1, \alpha 5 \beta 3$, and $\alpha 4 \beta 1$, contribute to SMC migration and synthetic phenotype. Previously, it was shown that $\alpha 9 \beta 1$ is expressed by vascular SMCs (7). However, no prior studies have examined how lack of $\alpha 9 \beta 1$ affects SMC proliferation and neointimal hyperplasia. We demonstrated that cultured SMCs from the human coronary artery upregulated $\alpha 9$ following stimulation with PDGF-BB, which was associated with phenotypic switching from contractile to synthetic state. We speculate that phenotypic switching of SMCs from low to high $\alpha 9$ expression may reduce protective homeostasis in these cells, thus resulting in SMC proliferation and neointimal hyperplasia. Indeed, targeting $\alpha 9$ in stimulated human coronary SMCs, using anti- $\alpha 9$ antibody, suppressed synthetic phenotype and inhibited SMC proliferation and migration. The anti- $\alpha 9$ antibody may have off-target side effects. To provide definitive evidence, we generated $\alpha 9$-deficient mice. $\beta 1$ is the only known subunit of $\alpha 9$; therefore, lack of $\alpha 9$ should completely inhibit $\alpha 9 \beta 1$ signaling. We did not target $\beta 1$ in addition to $\alpha 9$ because $\beta 1$ binds to several other subunits, including $\alpha 4$ and $\alpha 5$ that may confound our findings. In line with these observations, we found that genetic ablation of $\alpha 9$ in murine SMCs suppressed synthetic phenotype and reduced proliferation and migration in vitro and inhibited neointimal hyperplasia in vivo.

We also investigated the molecular mechanism by which integrin $\alpha 9 \beta 1$ promotes SMC proliferation, migration, and neointimal hyperplasia. We found that therapeutic targeting of $\alpha 9$ in human coronary artery SMCs or genetic deletion of $\alpha 9$ significantly reduced FAK, Src, and MAPK activation, which are known to regulate a wide range of cellular responses, including proliferation and migration. One of the signaling pathways downstream of integrin activation is the canonical Wnt/ $\beta$-catenin pathway (26). We found that the lack of $\alpha 9$ in SMCs results in reduced level of nonphosphorylated $\beta$-catenin, which is considered the active form of $\beta$-catenin. GSK3 $\beta$ phosphorylates $\beta$-catenin and promotes its ubiquitylation and subsequent proteasomal degradation (15). Integrin and growth factor receptors can activate GSK3 $\beta$, and the lack of integrin $\alpha 9$ in a cancer cell is associated with higher GSK3 $\beta$ activity (26). In line with these findings, we found reduced p-GSK3 $\beta$ in SMCs lacking $\alpha 9$. Nuclear localized $\beta$-catenin interacts with the TCF/LEF family of transcription factors and promotes gene expression. We found that stimulated SMCs lacking $\alpha 9$ had reduced nuclear $\beta$-catenin levels associated with decreased transcriptional activation of MMP-2, MMP-9, cyclin D1, and c-Myc. Together, these findings suggest that $\alpha 9$ may contribute to SMC proliferation and migration by upregulating the $\beta$-catenin pathway. 
A
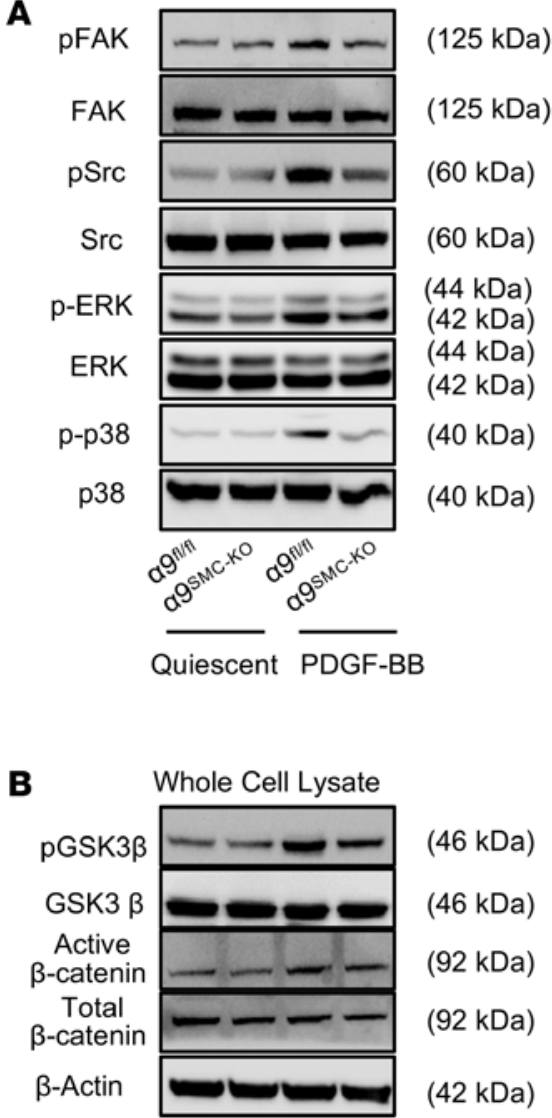

(46 kDa)

$(46 \mathrm{kDa})$

(92 kDa)

$(92 \mathrm{kDa})$

$(42 \mathrm{kDa})$

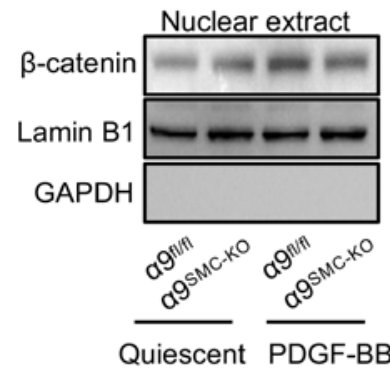

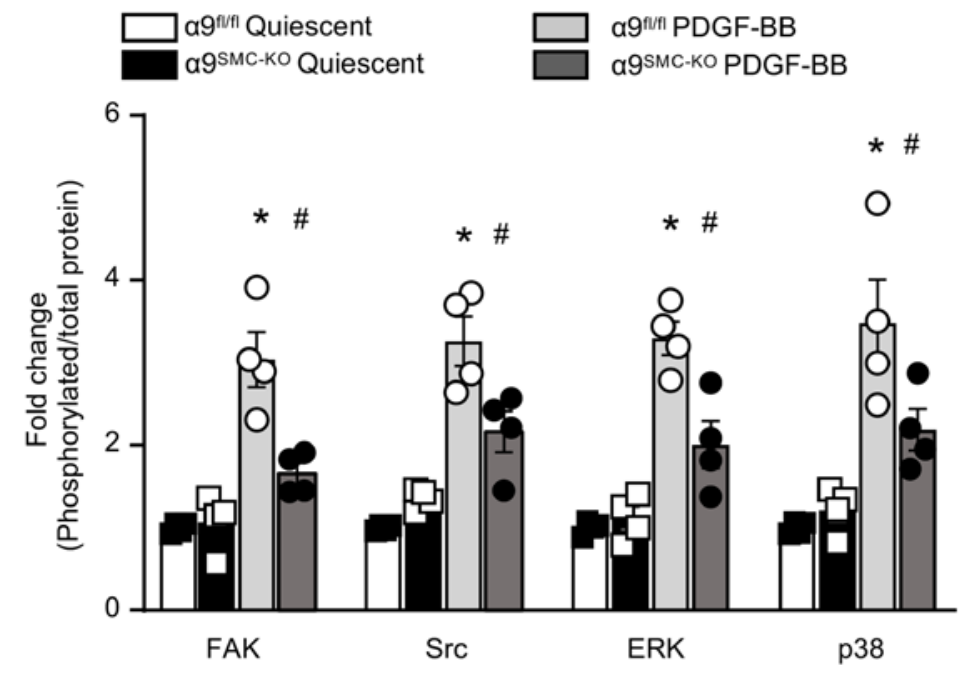

$\begin{array}{ll}\square \text { a9f/fl Quiescent } & \square \text { a9f/fl PDGF-BB } \\ \text { a9smc-ko Quiescent } & \text { a9smc-ko PDGF-BB }\end{array}$

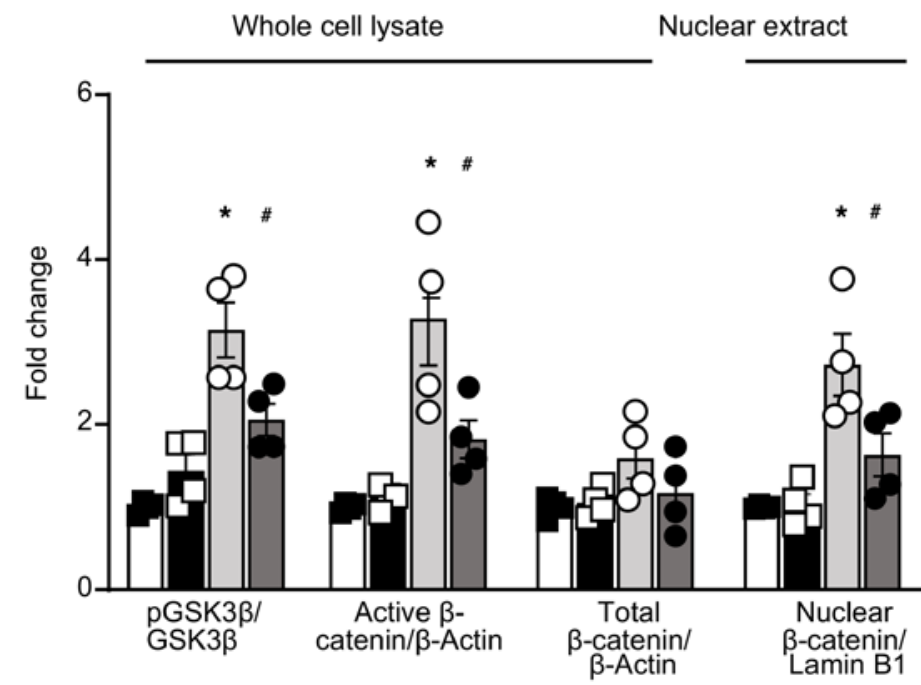

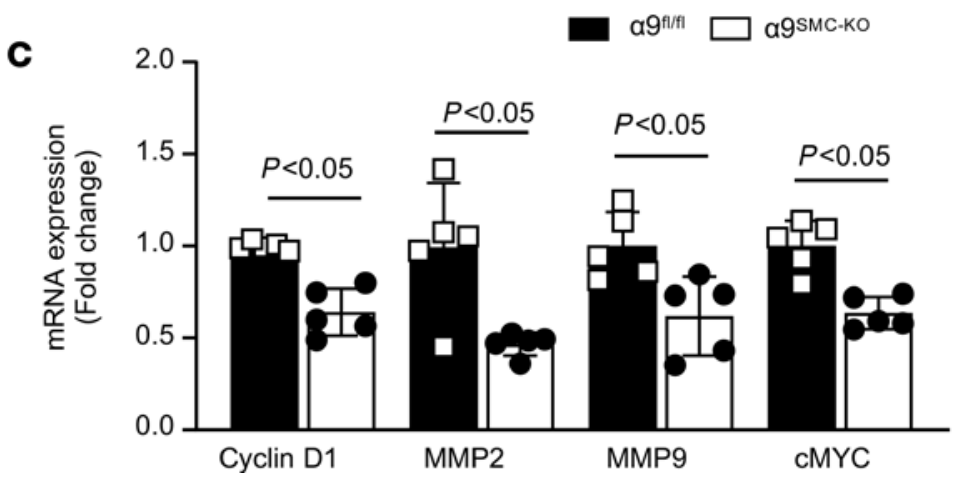

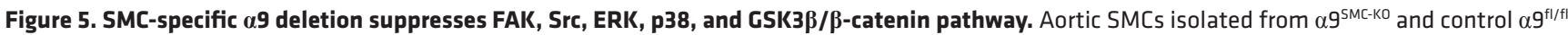
mice were serum-starved for 48 hours. (A) Representative Western blots and densitometric analysis of FAK, Src, ERK1/2, p38, and $\beta$-actin after 30 minutes of PDGF-BB stimulation $(n=4)$. (B) Representative Western blots and densitometric analysis of GSK3 $\beta$, $\beta$-catenin, and $\beta$-actin after 30 minutes of PDGF-BB stimulation $(n=4)$. Nuclear extracts were prepared after 6 hours of PDGF-BB stimulation. $\beta$-Catenin and Lamin B1 were detected by immunoblotting $(n=4)$. (C) Real-time quantitative PCR analysis of cyclin D1, MMP-2, MMP-9, and c-Myc $(n=5)$ in SMCs stimulated with PDCF-BB $(20 \mathrm{ng} / \mathrm{mL})$ for 24 hours. Values are expressed as mean \pm SEM. Statistical analysis: (A and B) 2-way ANOVA followed by uncorrected Fisher's LSD test. ${ }^{*} P<0.05$ versus $\alpha 9^{f / / f l}$ quiescent SMCs, ${ }^{\#} P<0.05$ vs $\alpha 9^{f \mid / f l}$ PDCF-BB-treated SMCs. (C) Unpaired 2-tailed Student's $t$ test. 
Integrins play a crucial role in SMC biology by binding to the extracellular matrix (ECM) with the SMC actin cytoskeleton. Integrin $\alpha 9 \beta 1$ binds to several components of ECM proteins, including osteopontin, tenascin-C, and cellular fibronectin-containing EDA (Fn-EDA). Additionally, $\alpha 9 \beta 1$ and its matrix protein ligands associate and synergize signaling from several growth factors, including PDGF-BB, to promote cell adhesion motility $(4,27,28)$. Integrins such as $\alpha v \beta 3, \alpha 5 \beta 1$, and $\alpha 8 \beta 1$ bind to ECM containing an RGD (Arg-Gly-Asp) motif including fibronectin and vitronectin. Unlike other integrins that recognize RGD sequence, $\alpha 9 \beta 1$ recognizes several non-RGD sequences, including SVVYGLR in osteopontin (29), AEIDGIEL in tenascin-C (30), and PEDGIHELFP in Fn-EDA (21). The first evidence that plasma fibronectin (which does not contain extra domain A) plays a significant role in phenotypic switching via integrins was demonstrated by Hedin and Thyberg (31). However, integrin $\alpha 9 \beta 1$ is a ligand for cellular Fn-EDA but not plasma fibronectin or cellular Fn-EDB. We have previously shown that PDGF-BB upregulates cellular Fn-EDA expression levels in stimulated SMCs and promotes phenotype switching, proliferation, and migration through integrins (not recognized by RGD sequence) and TLR4-dependent pathways (13). Notably, cellular Fn-EDA was more potent than plasma fibronectin in promoting phenotypic switching and the proliferation of SMCs. Integrin $\alpha 5 \beta 1$ is another ligand for fibronectin and is known to play a role in SMC proliferation; however, $\alpha 5 \beta 1$ recognizes RGD sequence in fibronectin. Because $\alpha 9 \beta 1$ recognizes non-RGD sequences and binds explicitly to Fn-EDA, we hypothesized that $\alpha 9 \beta 1$ might contribute to SMC phenotypic switching and proliferation via Fn-EDA. Indeed, we found that Fn-EDA partially contributes to integrin $\alpha 9$-mediated SMC phenotypic switching and proliferation. We speculate that other ECM proteins, including osteopontin and tenascin-C, may contribute to integrin $\alpha 9$-mediated SMC phenotypic switching and proliferation in addition to Fn-EDA. A summary of the proposed mechanism is provided in Figure 7E.

Antibody or oligonucleotide infusion or viral vectors have been widely used to inhibit the signaling pathways for the prevention of neointimal hyperplasia (32-36). Next, we evaluated whether injury-induced neointimal hyperplasia could be reduced by blocking $\alpha 9 \beta 1$ signaling. Herein, we demonstrate that targeting $\alpha 9 \beta 1$ signaling using anti-integrin $\alpha 9$ antibody (55A2C) reduced neointimal hyperplasia in WT mice by limiting SMC proliferation and migration. Anti-integrin $\alpha 9$ antibody is known to inhibit the binding of $\alpha 9 / \mathrm{NIH} 3 \mathrm{~T} 3$ cells to the synthetic peptides AEIDGIEL, the sequence similar to the EDGIHEL sequence present in the EDA of cellular fibronectin (21). Based on these observations, we speculate that the beneficial effects of the anti-integrin $\alpha 9$ antibody on neointimal hyperplasia might be partly due to the inhibition of integrin $\alpha 9 \beta 1$ binding to cellular Fn-EDA. Notably, other cells, including neutrophils, hepatocytes, endothelial cells, and epithelial cells, express $\alpha 9$, and the possibility that other cell types may contribute to neointimal hyperplasia cannot be ruled out. Further studies are required to determine the role of integrin $\alpha 9$ in other cell types in neointimal hyperplasia.

A particular strength of our study is that we generated SMC-specific deficient mice to provide conclusive evidence that integrin $\alpha 9 \beta 1$ regulates multiple aspects of SMC biology. In WT mice, we demonstrated that targeting $\alpha 9$ by infusing 55A2C antibody reduced neointimal hyperplasia at 28 days. Despite its strength, our study has limitations. We used a wire injury model that partially mimics balloon angioplasty or in-stent restenosis. Future studies are warranted to confirm the efficacy of targeting integrin $\alpha 9$ in in-stent models. We suggest that therapeutically targeting integrin $\alpha 9$ may be beneficial in patients to reduce intimal hyperplasia during both open and endovascular procedures.

\section{Methods}

Antibodies. A detailed list of antibodies with catalog number and company is provided in Supplemental Table 2. Animals. The $\alpha 9^{\mathrm{fl} / \mathrm{l}}$ mouse strain has been described previously (37) (provided by Matthew G. Vander Heiden, MIT, Boston, Massachusetts, USA). To generate SMC-specific $\alpha 9$-deficient mice ( $\alpha 9^{\mathrm{f} / \mathrm{fl}} \mathrm{SM} 22 \alpha \mathrm{Cre}^{+} \mathrm{Apoe}^{-/-}$), $\alpha 9^{\mathrm{fl} / \mathrm{fl}} \mathrm{Apoe}^{-/-}$mice (generated in-house) were crossed with SM22 $\alpha \mathrm{Cre}^{+} \mathrm{Apoe}^{-/-}$mice (provided by Isabella Grumbach, University of Iowa, Iowa City, Iowa, USA).

The SM22 $\alpha \mathrm{Cre}^{+}$transgenic mice expressed Cre recombinase under the control of mouse SMC protein $22 \alpha$ (or transgelin) promoter. Littermates $\left(\alpha 9^{\mathrm{fl} / \mathrm{fl}} \mathrm{SM} 22 \alpha \mathrm{Cre}^{-} \mathrm{Apoe}^{-/-}\right.$) were used as controls. Fn-EDA ${ }^{-/} \mathrm{Apoe}^{-/-}$ mice (generated in-house) have been described previously (38) (Fn-EDA ${ }^{-1-}$ mice provided by Andrés F. Muro, ICGEB, Trieste, Italy). All the mouse strains are on a C57BL/6J background (backcrossed $>15$ times). Mice were genotyped by PCR according to protocols from The Jackson Laboratory and as described previously (38). C57BL/6J mice (7-8 weeks old, males) were procured from The Jackson Laboratory. Mice were kept in standard animal housing conditions with controlled temperature and humidity and had ad libitum access to standard chow diet and water. All the experiments involving animals were performed according to protocols 
A BrdU/a-SMA/Hoechst

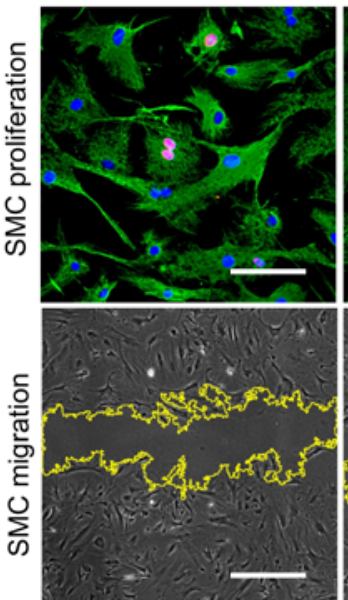

EDA (-) peptide
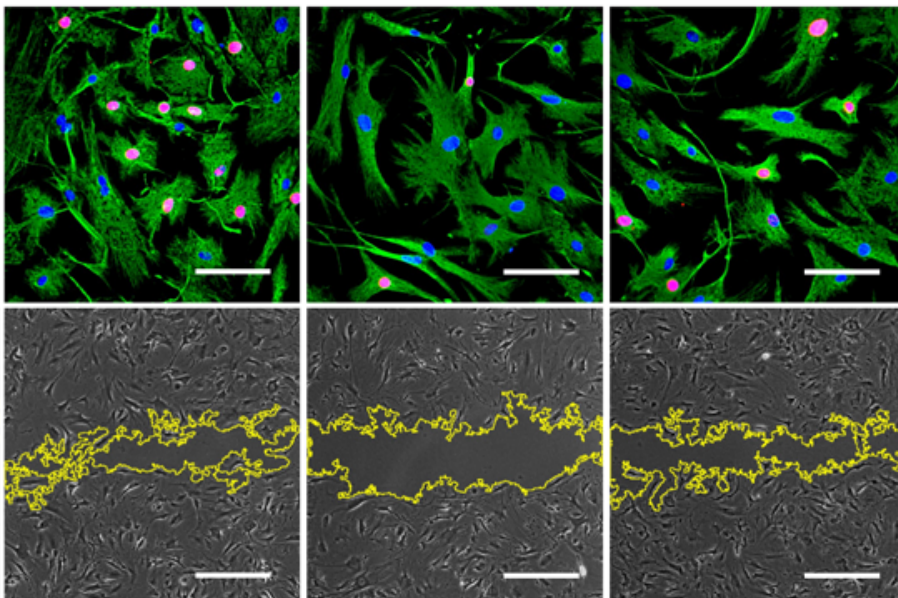

EDA (-) peptide

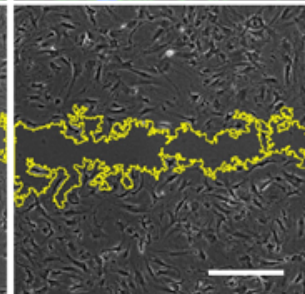

EDA (+) peptide
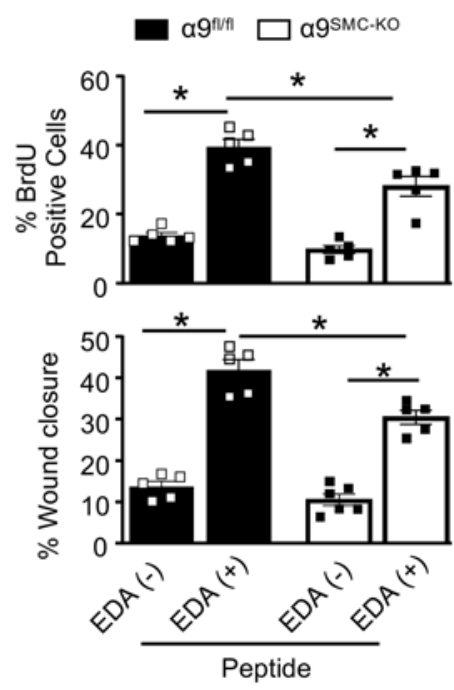

B

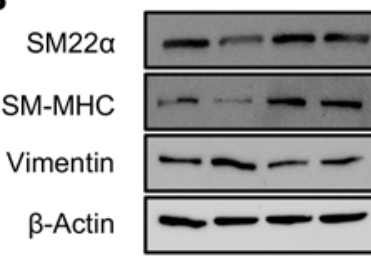

(22 kDa)

(227 kDa)

(54 kDa)

(42 kDa)

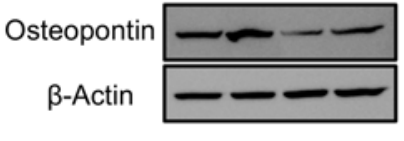

(60 kDa)

(42 kDa)

EDA (-)

peptide

EDA (+)

peptide

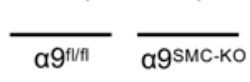

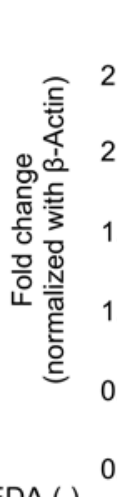

EDA (-) peptide

EDA (+) peptide

a9smc-ko
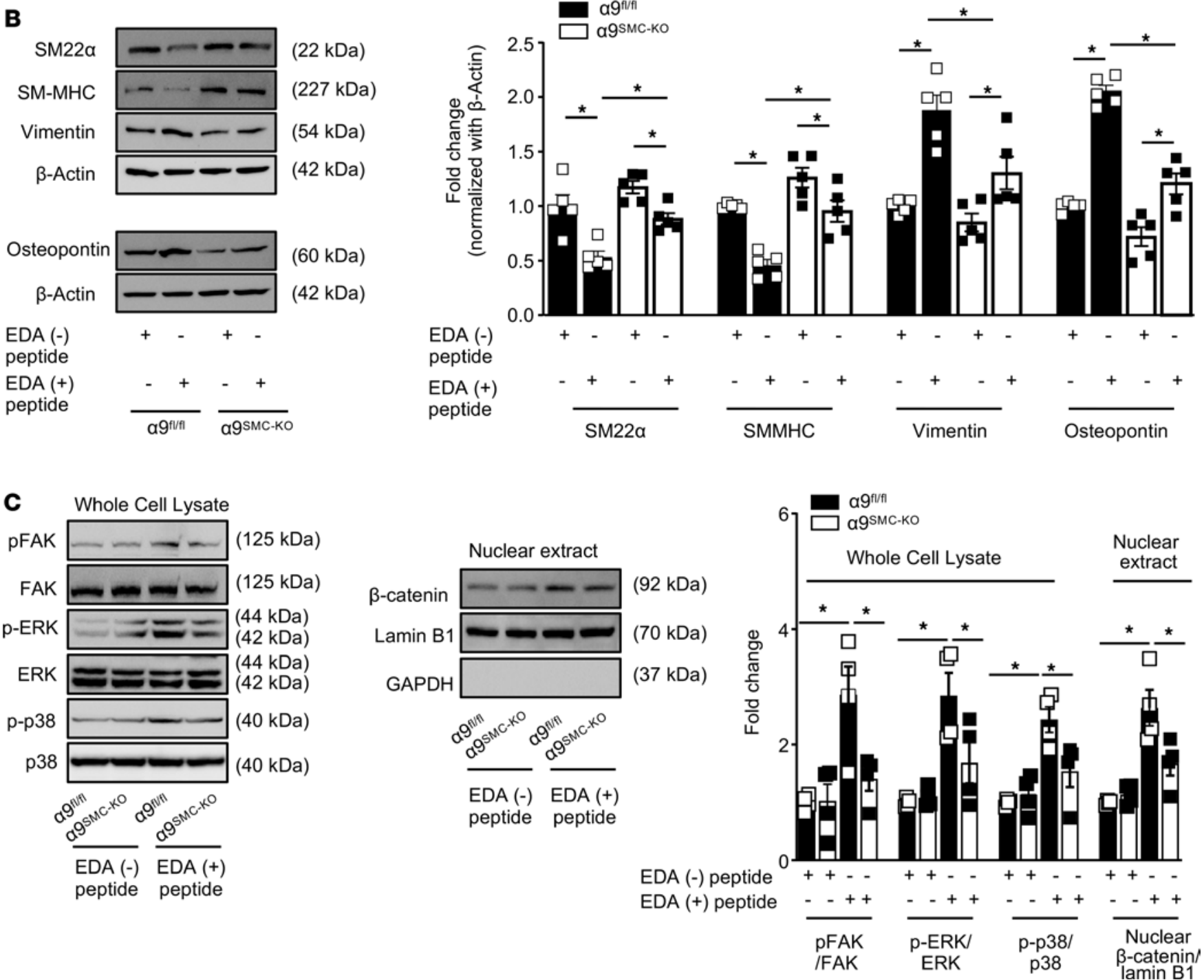

Figure 6. Fn-EDA partially contributes to $\alpha \mathbf{9}$-mediated SMC proliferation and migration. Quiescent SMCs were exposed to $10 \mu \mathrm{g} / \mathrm{mL}$ of recombinant peptides containing or lacking EDA. (A) The upper left panels show representative images of BrdU-positive cells (red) costained with $\alpha$ SMA (green) and Hoechst (blue) analyzed 24 hours after EDA peptide treatment. Scale bar: $50 \mu \mathrm{m}$. Lower left panels show representative phase-contrast images of SMC 
migration in the scratch assay analyzed 24 hours after EDA peptide treatment. Scale bar: $500 \mu \mathrm{m}$. The right panels show quantification of BrdU-positive cells $(n=5)$ and migrated area $(n=5)$. (B) Cells were processed for Western blotting after 24 hours of EDA peptide treatment. Representative immunoblots and densitometric analysis of SM22 $\alpha$, SM-MHC, vimentin, osteopontin, and $\beta$-actin $(n=5)$. (C) Representative immunoblots and densitometric analysis of FAK, Src, ERK1/2, p38, GSK3 $\beta$, $\beta$-catenin, and $\beta$-actin after 30 minutes of PDGF-BB stimulation ( $n=4 /$ group). Nuclear extracts were prepared after 6 hours of PDGF-BB stimulation. $\beta$-Catenin and Lamin B1 were detected by immunoblotting ( $n=4$ /group). Values are expressed as mean \pm SEM. Statistical analysis: 2-way ANOVA followed by Fisher's LSD test. ${ }^{*} P<0.05$.

approved by the University of Iowa Animal Care and Use Committee according to the current Animal Research: Reporting of In Vivo Experiment guidelines (https://www.nc3rs.org.uk/revision-arrive-guidelines).

Mouse carotid artery injury model of neointimal hyperplasia. Carotid artery wire injury was performed by using a guidewire (0.015-inch diameter, C-SF-15-15, Cook Medical) as described previously (39). Briefly, the left common carotid artery was exposed by blunted dissection. The external carotid artery was exposed, and an incision was made through which the guidewire was advanced $1 \mathrm{~cm}$ toward the aorta. The wire was moved back and forth 3 times to ensure endothelial denudation. The wire was retrieved, and the external carotid artery was ligated with a 7-0 vicryl suture. The wound was closed with a subcuticular 7-0 vicryl suture. Animals were allowed to recover before returning to the cages. At 28 days after injury, the animals were euthanized, and carotid arteries ( $\sim 5.0 \mathrm{~mm}$ from injury site) were carefully harvested and processed for cryostat sectioning for subsequent analysis. Some animals received 2 subcutaneous doses of BrdU (Millipore Sigma, $100 \mathrm{mg} / \mathrm{kg}$ ) at 12 hours and 1 hour prior to the mice being sacrificed (40).

Injection of anti-integrin $\alpha 9$ antibody. Mice were randomly assigned and infused with either anti- $\alpha 9$ antibody (55A2C, $200 \mu \mathrm{g} / \mathrm{mouse}$, provided as a gift from Gene Techno Science Co Ltd) or control Ig isotype (200 $\mu \mathrm{g} /$ mouse, Rockland, catalog 610-4107-0500) intravenously (41, 42), 60 minutes prior to carotid artery wire injury.

Histology and morphometric analysis. To quantify intimal hyperplasia, serial $5 \mu \mathrm{m}$ cross sections from injured and noninjured arterial segments were stained with Verhoeff's van Gieson stain as described (43). Bright-field images were acquired using an Olympus BX51 microscope equipped with a $\times 20$ objective. Circumferences of the external and internal elastic lamina (EEL, IEL) and the lumen were traced on the stained sections (44). The intimal area was determined by subtracting the lumen area from the area circumscribed by the IEL traced on stained sections, whereas medial area was defined as the area between an EEL and an IEL (45). Intima/media area ratio was calculated by dividing the intimal area by medial area (46). Calculations were made using the mean value of 4-6 sections (each approximately $100 \mu \mathrm{m}$ apart) from each mouse artery. NIH ImageJ software was used for quantification.

Human and mouse SMC culture and treatment. Human primary coronary artery SMCs were obtained from Lonza (catalog number CC-2583) and cultured in smooth muscle basal medium (Lonza; catalog number CC-3181) containing recombinant human FGF basic $(5 \mathrm{ng} / \mathrm{mL})$, insulin $(5 \mu \mathrm{g} / \mathrm{mL}), \mathrm{EGF}(5 \mathrm{ng} / \mathrm{mL})$, and FBS (5\%). Primary murine SMCs were isolated from the thoracic aorta by enzymatic digestion technique as described previously (47). Briefly, the thoracic aorta was dissected and cleaned of adventitial tissue and incubated with $1.0 \mathrm{mg} / \mathrm{mL}$ collagenase in DMEM at $37^{\circ} \mathrm{C}$ for 90 minutes. The cell suspension was passed through a $70 \mu \mathrm{m}$ restrainer, washed, and cultured in DMEM with 10\% FBS. Cells were maintained in a $95 \%$ air and $5 \% \mathrm{CO}_{2}$ humidified incubator at $37^{\circ} \mathrm{C}$ for 7 days, trypsinized with $0.25 \%$ trypsin-EDTA, and subcultured. All SMCs used for experiments were between the third and fifth passages.

EDA-containing and EDA-lacking recombinant peptides. Fibronectin sequences containing [EDA (+) peptide] or lacking [EDA (-) peptide] the EDA segment were generated from full-length cellular fibronectin $(\mathrm{cFn})$ and full-length plasma fibronectin ( $\mathrm{pFn}$ ) cDNAs, respectively, as described before (48), and were used at a concentration of $10 \mu \mathrm{g} / \mathrm{mL}$.

Immunocytochemistry. Immunostaining was performed in sections from mouse carotid arteries and isolated mouse and human SMCs. Mouse arterial sections or cells were fixed in 4\% paraformaldehyde (PFA, in PBS) for 10 minutes and permeabilized with $0.2 \%$ Triton X-100 (in PBS). Nonspecific binding was blocked for 1 hour with $5 \%$ serum from the species in which the secondary antibody was raised. Samples were incubated overnight at $4^{\circ} \mathrm{C}$ with antibodies specific for integrin $\alpha 9$ (1:100, Abcam), vimentin (1:100, Abcam), osteopontin (1:100, Abcam), SM-MHC (1:100, Abcam), SM22 $\alpha$ (1:100, Abcam), and aSMA (1:200, MilliporeSigma) overnight at $4^{\circ} \mathrm{C}$. Isotype control Ig was used as a negative control. After overnight incubation, cells/slides were washed with PBS and labeled with appropriate secondary antibodies (Alexa Fluor 488 or Alexa Fluor 546 at 1:400, Abcam) for 1 hour at room temperature. Nuclei were stained with Hoechst (5 $\mu \mathrm{g} / \mathrm{mL}$ ) before mounting. Nikon Eclipse Ti-U inverted fluorescence microscope equipped with a $\times 20 / 0.8$ 
A BrdU/a-SMA/Hoechst

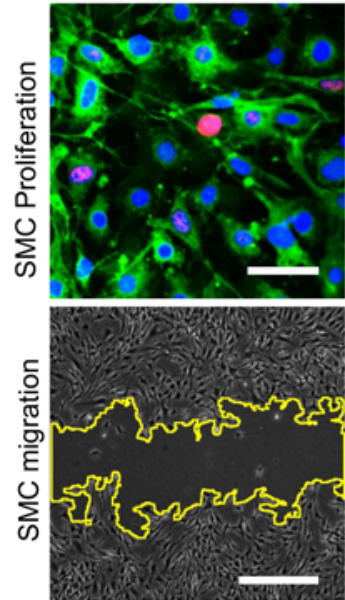

Quiescent
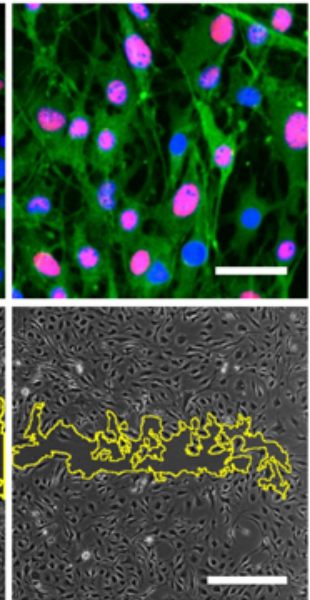

PDGF-BB+

Control-Ig
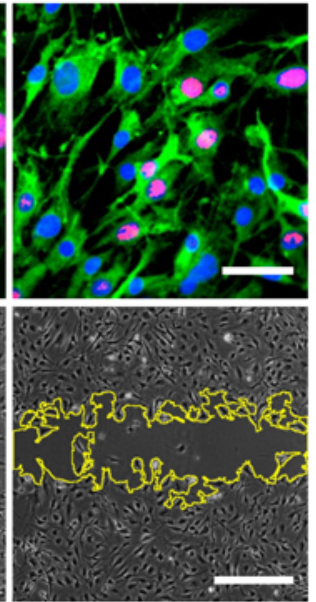

PDGF-BB +

Anti a9-lg
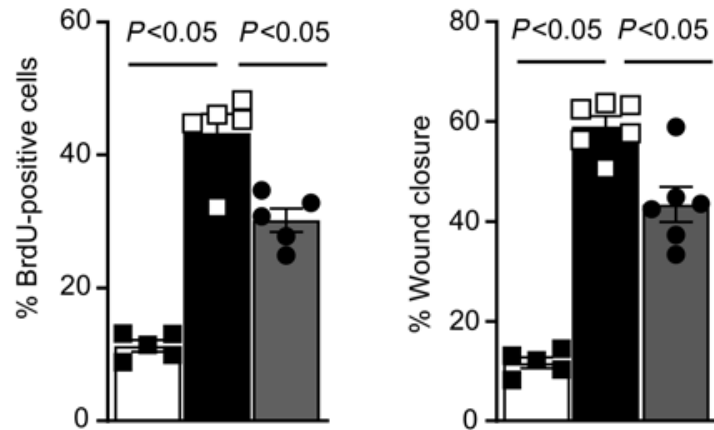

B

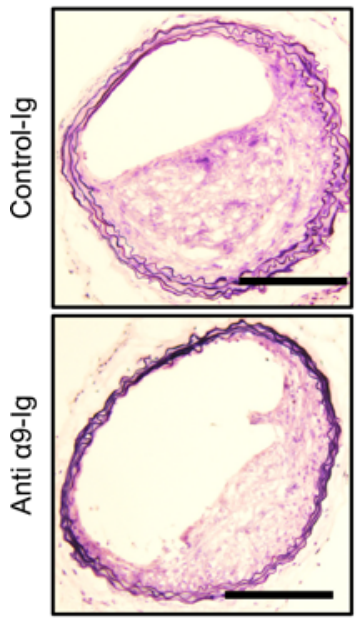

Injured carotid (28 days)
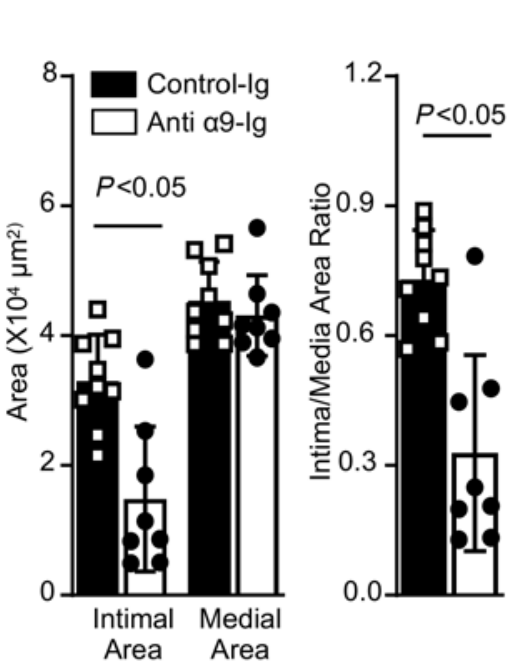

C BrdU/aSmA/Hoechst
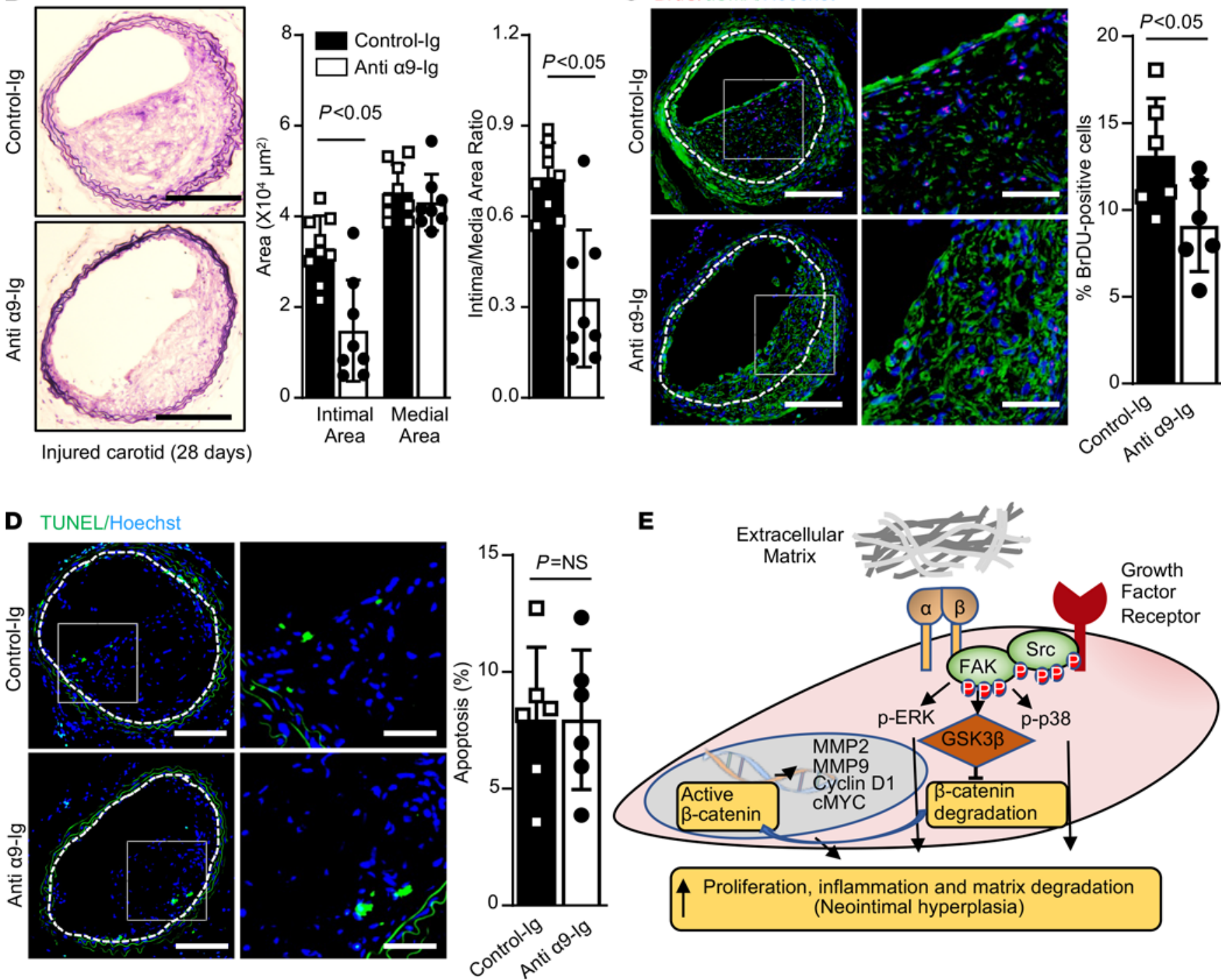

E

Extracellular Matrix

\section{Growth}

Factor Receptor
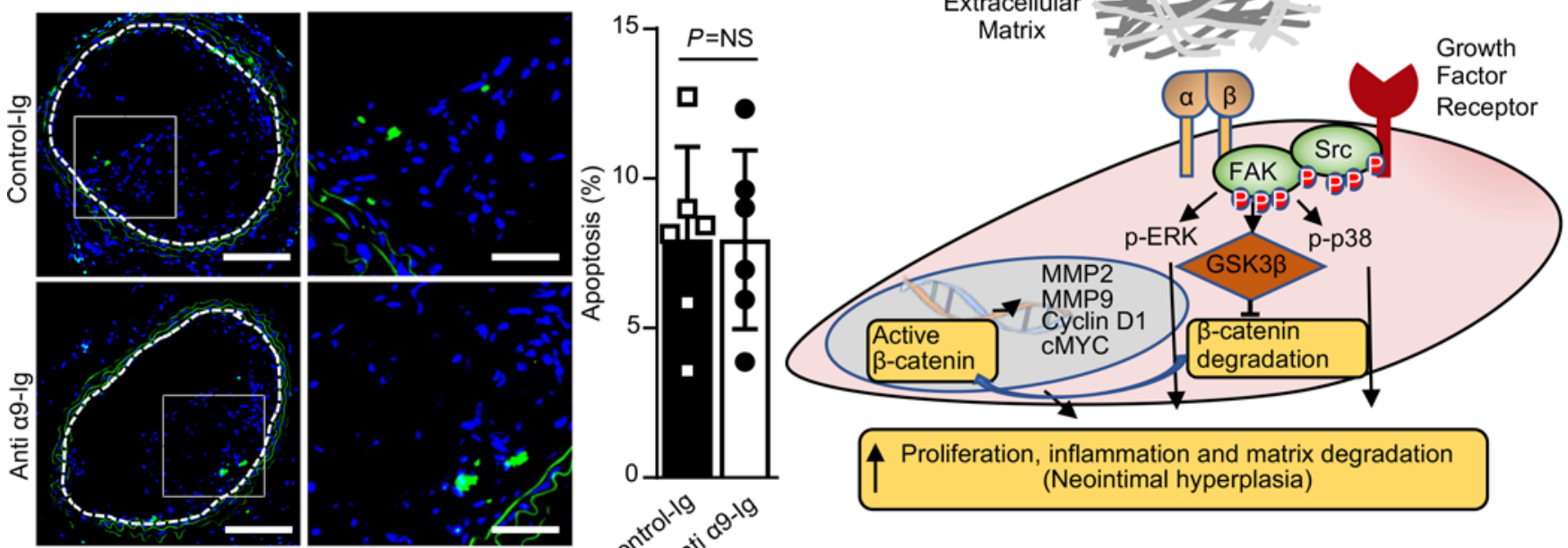

Figure 7. Infusion of anti-integrin $\alpha 9$ antibody reduces injury-induced neointimal hyperplasia in WT mice. Serum-starved SMCs from WT mice were pretreated with murine specific anti- $\alpha 9$ blocking antibody (clone $55 \mathrm{~A} 2 \mathrm{C}, 10 \mu \mathrm{g} / \mathrm{mL}$ ) for 60 minutes and then stimulated with or without PDCF-BB for 24 hours. (A) The top panels show representative BrdU-positive (red) cells costained with $\alpha$ SMA (green) and Hoechst (blue). Scale bars: $50 \mu \mathrm{m}$. The bottom panels show representative phase-contrast images of SMC migration in the scratch assay. Scale bars: $500 \mu \mathrm{m}$. The right panel shows the quantification of BrdU-positive 
cells to the total number of cells $(n=5)$ and migrated area $(n=6)$. (B-D) Male WT mice were treated with 55A2C (200 $\mu \mathrm{g} / \mathrm{mouse})$ or control lg. Wire injury was performed in the carotid artery after 60 minutes, and arteries were harvested after 28 days. (B) The left panels show representative photomicrographs of Verhoeff's van Gieson-stained carotid artery sections. Scale bars: $200 \mu \mathrm{m}$. The right panels show quantification of intimal area, medial area, and a ratio of intimal to medial area. Each dot represents a single mouse ( $n=8-9 /$ group). (C) The left panels show representative BrdU-positive cells (red) counterstained with aSMA (green). Nuclei are counterstained with Hoechst (blue). The right panel shows the quantification of percentage BrdU-positive cells ( $n=6$ ). Scale bars: $200 \mu \mathrm{m}$. (D) The left panels show representative TUNEL-positive cells (green) counterstained with Hoechst (blue). The right panel shows the quantification of TUNEL-positive cells $(n=6)$. Scale bars: $200 \mu \mathrm{m}$. Values are represented as mean \pm SEM. Statistical analysis: (A) 1-way ANOVA with Bonferroni's post hoc test; (B-D) unpaired 2-tailed Student's $t$ test. ${ }^{*} P<0.05$ vs. quiescent or vehicle-treated (control lg) groups. (E) Schematic showing the mechanism by which SMC-specific integrin $\alpha 9$ mediates SMC proliferation, migration, and neointimal hyperplasia.

Plan Apo lens, cooled charge-coupled device camera, and NIS-Elements imaging software (Nikon) was used for imaging of SMCs. For arterial sections, images were taken using an Olympus BX51 fluorescence microscope. Images were acquired under identical imaging conditions. Mean fluorescence intensity was quantified using the ImageJ software, as previously described (49). Measurements were obtained from 1-2 different fields per murine section and 4-6 different fields for cultured SMCs.

BrdU incorporation assay. Human and mouse SMCs were serum-starved for 48 hours to induce quiescence. Cells were washed and stimulated with PDGF-BB $(20 \mathrm{ng} / \mathrm{mL}), \mathrm{cFn}(20 \mu \mathrm{g} / \mathrm{mL})$ or $\mathrm{pFn}(20 \mu \mathrm{g} / \mathrm{mL})$, and recombinant EDA-containing or EDA-lacking peptides $(10 \mu \mathrm{g} / \mathrm{mL})$ for 24 hours. Cells were incubated with $10 \mu \mathrm{M}$ BrdU for the last 12 hours of treatment (50). Carotid artery sections from BrdU-treated mice or cultured SMCs were fixed with 4\% PFA (in PBS). DNA hydrolysis was performed by treating the cells/sections with $2 \mathrm{M} \mathrm{HCl}$ for 20 minutes. Samples were subjected to immunofluorescence staining with a mouse monoclonal anti-BrdU antibody (1:200, Abcam) together with $\alpha$ SMA (1:200, MilliporeSigma) for 3 hours at $37^{\circ} \mathrm{C}$ and labeled with appropriate Alexa Fluor 488 and Alexa Fluor 546 secondary antibodies (1:400, Abcam). Nuclei were stained with Hoechst $(5 \mu \mathrm{g} / \mathrm{mL})$ before mounting. Images of the sections were acquired using an Olympus BX51 fluorescence microscope, and images of cells were acquired using the Nikon Eclipse Ti-U fluorescence microscope. The number of BrdU-positive cells in the neointima or the media and the total number of nuclei were determined using ImageJ software. The percentage of BrdU-positive cells was calculated as (number of BrdU-positive nuclei in the neointima or media/total number of nuclei) $\times 100$.

Migration assay. Cultured human and mouse aortic SMCs were seeded in 6-well culture plates and were grown in DMEM until confluence. Cells were serum-starved for 48 hours, and a manual scratch was created with a $200 \mu \mathrm{L}$ pipette tip to create a cell-free zone. Cells were washed with PBS to remove nonadherent cells and incubated in a serum-free medium. Images of the scratched area were taken immediately using a Nikon Eclipse Ti-U phase-contrast microscope. Cells were then stimulated with PDGF-BB (20 ng/mL) and recombinant EDA-containing or EDA-lacking peptides $(10 \mu \mathrm{g} / \mathrm{mL})$ for 24 hours. The second set of images were taken after 24 hours to measure SMC migration over the scratched area. The cell-free area for each well was measured using the ImageJ software and the MRI wound healing tool plug-in (51). The percentage of wound closure was calculated as the difference of denuded area between 0 and 12 or 24 hours to the denuded area at 0 hours.

Western blotting. Cell or tissue proteins were extracted with RIPA lysis buffer containing protease inhibitor cocktail. For nuclear extraction experiments, nuclear and cytoplasmic fractions were isolated using the NE-PER Nuclear and Cytoplasmic Extraction kit (Invitrogen, Thermo Fisher Scientific), following the manufacturer's instructions. Equal amounts of protein samples were resolved by SDS-PAGE and transferred to PVDF membranes by using a Bio-Rad Western blotting system. The membranes were blocked with 5\% BSA or $5 \%$ nonfat dry milk in Tris-buffered saline containing $0.1 \%$ Tween 20 for 1 hour at room temperature. The membrane was then incubated overnight at $4^{\circ} \mathrm{C}$ with primary antibodies against integrin $\alpha 9, \mathrm{p}$-FAK (Tyr397), FAK, p-Src family (Tyr416), Src, p-p44/42 MAPK (Erk1/2) (Thr202/Tyr204), p44/42 MAPK (Erk1/2), p-GSK3 $\beta$, GSK3 $\beta$, active $\beta$-catenin, total $\beta$-catenin, and $\beta$-actin. Following incubation with appropriate horseradish peroxidase-conjugated secondary antibodies, the blots were visualized and imaged using ECL Plus Kit (MilliporeSigma) and ChemiDoc Imaging System (Bio-Rad). Densitometric analysis of the gels was done using ImageJ software. The proteins were normalized with $\beta$-actin (MilliporeSigma) and respective total protein content. See complete unedited blots in the supplemental material.

TUNEL staining. Apoptotic cells within the carotid artery sections were visualized using In Situ Cell Death Detection Kit, Fluorescein (Roche, 11684809910), as per manufacturer's instructions. Briefly, arterial sections were fixed, permeabilized with $0.2 \%$ Triton X-100, and subsequently incubated with TUNEL reaction mixture (Roche) for 60 minutes at $37^{\circ} \mathrm{C}$ in a humidified chamber. Nuclei were stained with Hoechst $(5 \mu \mathrm{g} / \mathrm{mL})$. 
Sections were examined under a fluorescence microscope (Olympus BX51). The percentage of apoptotic cells to the total number of cells was quantified using ImageJ software as described previously $(52,53)$.

$R N A$ isolation and quantitative real-time PCR analysis. Total cellular RNA was isolated from murine SMCs using an RNeasy Kit (QIAGEN, 74104) as per the manufacturer's instructions. cDNA was prepared using iScript Reverse Transcription Supermix (Bio-Rad, 1708840). PCR amplification of the cDNA (100 ng) was performed with the Applied Biosystems 7900HT Fast Real-Time PCR machine (total volume $20 \mu \mathrm{L}$, Thermo Fisher Scientific) at the University of Iowa's Iowa Institute of Human Genetics Genomics Division. Levels of mRNA were normalized by GAPDH (as a loading control) and calculated according to the comparative threshold cycle ( $\triangle \mathrm{Ct}$ value). Primers for quantitative real-time PCR for the indicated genes are included in Supplemental Table 3.

Statistics. Data are expressed as mean \pm SEM. The number of animals in each group was based on power calculations for the primary parameter (neointimal area) with mean differences and SDs taken from pilot data at power $80 \%$ with an $\alpha$ of 0.05 . GraphPad Prism (version 8.4.1) was used for statistical analysis. The Shapiro-Wilk test was used to check normality, and Bartlett's test was used to check equal variance. The statistical significance of the difference between means was assessed using the unpaired 2-tailed Student's $t$ test (for the comparison of 2 groups), by 1-way ANOVA followed by Bonferroni's multiple comparisons tests (parametric data of more than 2 groups), by Kruskal-Wallis test followed by uncorrected Dunn's test (nonparametric data of more than 2 groups), or by 2-way ANOVA followed by uncorrected Fisher's LSD test. $P<0.05$ was considered significant.

Study approval. The University of Iowa Animal Care and Use Committee approved all procedures, and studies were performed according to the current Animal Research: Reporting of In Vivo Experiment guidelines (https://www.nc3rs.org.uk/revision-arrive-guidelines).

\section{Author contributions}

$\mathrm{MJ}$ and $\mathrm{AKC}$ conceived the study; $\mathrm{MJ}, \mathrm{RD}, \mathrm{PD}$, and $\mathrm{ND}$ analyzed data; $\mathrm{MJ}, \mathrm{RD}, \mathrm{PD}$, and ND investigated; SK provided reagents; MJ and AKC wrote the original draft; and all authors reviewed and edited the draft.

\section{Acknowledgments}

Andrés F. Muro provided EDA-containing and EDA-lacking recombinant peptides. The AKC lab is supported by grants from the NIH (R35HL139926, R01NS109910, U01NS113388) and by Established Investigator Award 18EIA33900009 from American Heart Association.

Address correspondence to: Manish Jain or Anil K. Chauhan, Department of Internal Medicine, 3120 Medical Labs, University of Iowa, Iowa City, Iowa 52242, USA. Phone: 319.335.6525; Email: manish-jain@ uiowa.edu (MJ); Email: anil-chauhan@uiowa.edu (AKC).

1. Alfonso F, et al. Current treatment of in-stent restenosis. J Am Coll Cardiol. 2014;63(24):2659-2673.

2. Aoki J, Tanabe K. Mechanisms of drug-eluting stent restenosis. Cardiovasc Interv Ther. 2021;36(1):23-29.

3. Shlofmitz E, et al. Restenosis of drug-eluting stents: a new classification system based on disease mechanism to guide treatment and state-of-the-art review. Circ Cardiovasc Interv. 2019;12(8):e007023.

4. Gupta SK, Vlahakis NE. Integrin alpha9beta1: unique signaling pathways reveal diverse biological roles. Cell Adh Migr. 2010;4(2):194-198.

5. Barczyk M, et al. Integrins. Cell Tissue Res. 2010;339(1):269-280

6. Kon S, Uede T. The role of $\alpha 9 \beta 1$ integrin and its ligands in the development of autoimmune diseases. J Cell Commun Signal. 2018;12(1):333-342.

7. Palmer EL, et al. Sequence and tissue distribution of the integrin alpha 9 subunit, a novel partner of beta 1 that is widely distributed in epithelia and muscle. J Cell Biol. 1993;123(5):1289-1297.

8. Singh P, et al. The spatial and temporal expression patterns of integrin alpha9beta1 and one of its ligands, the EIIIA segment of fibronectin, in cutaneous wound healing. J Invest Dermatol. 2004;123(6):1176-1181.

9. Paudel B, et al. Analysis of clinical factors affecting the restenosis following percutaneous coronary intervention. Nepal Med Coll J. 2005;7(2):101-106.

10. Wildgruber $\mathrm{M}$, et al. Increased intermediate $\mathrm{CD} 14^{++} \mathrm{CD} 16^{++}$monocyte subset levels associate with restenosis after peripheral percutaneous transluminal angioplasty. Atherosclerosis. 2016;253:128-134.

11. Nakazawa G, et al. The pathology of neoatherosclerosis in human coronary implants bare-metal and drug-eluting stents. $J$ Am Coll Cardiol. 2011;57(11):1314-1322.

12. Ahlen K, et al. Platelet-derived growth factor-BB modulates membrane mobility of beta1 integrins. Biochem Biophys Res Commun. 2004;314(1):89-96. 
13. Jain M, et al. Smooth muscle cell-specific fibronectin-EDA mediates phenotypic switching and neointimal hyperplasia. J Clin Invest. 2020;130(1):295-314.

14. Gupta SK, Vlahakis NE. Integrin alpha9beta1 mediates enhanced cell migration through nitric oxide synthase activity regulated by Src tyrosine kinase. J Cell Sci. 2009;122(pt 12):2043-2054.

15. Behrens J, et al. Functional interaction of an axin homolog, conductin, with beta-catenin, APC, and GSK3beta. Science. 1998;280(5363):596-599.

16. Takahashi J, et al. PDGF-dependent $\beta$-catenin activation is associated with abnormal pulmonary artery smooth muscle cell proliferation in pulmonary arterial hypertension. FEBS Lett. 2016;590(1):101-109.

17. Chen $\mathrm{C}$, et al. The Integrin alpha9beta1 contributes to granulopoiesis by enhancing granulocyte colony-stimulating factor receptor signaling. Immunity. 2006;25(6):895-906.

18. Wu B, et al. Wnt signaling induces matrix metalloproteinase expression and regulates $\mathrm{T}$ cell transmigration. Immunity. 2007;26(2):227-239

19. Yang W, et al. PKM2 phosphorylates histone H3 and promotes gene transcription and tumorigenesis. Cell. 2012;150(4):685-696

20. Yang W, et al. ERK1/2-dependent phosphorylation and nuclear translocation of PKM2 promotes the Warburg effect. Nat Cell Biol. 2012;14(12):1295-1304.

21. Liao YF, et al. The EIIIA segment of fibronectin is a ligand for integrins alpha 9beta 1 and alpha 4 beta 1 providing a novel mechanism for regulating cell adhesion by alternative splicing. J Biol Chem. 2002;277(17):14467-14474.

22. Ou J, et al. Fibronectin extra domain A (EDA) sustains CD133(+)/CD44(+) subpopulation of colorectal cancer cells. Stem Cell Res 2013;11(2):820-833.

23. Kanayama M, et al. Alpha9 integrin and its ligands constitute critical joint microenvironments for development of autoimmune arthritis. J Immunol. 2009;182(12):8015-8025.

24. Kon S, et al. A novel cryptic binding motif, LRSKSRSFQVSDEQY, in the C-terminal fragment of MMP-3/7-cleaved osteopontin as a novel ligand for $\alpha 9 \beta 1$ integrin is involved in the anti-type II collagen antibody-induced arthritis. PLoS One. 2014;9(12):e116210.

25. Nakamura-Ishizu A, et al. Extracellular matrix protein tenascin-C is required in the bone marrow microenvironment primed for hematopoietic regeneration. Blood. 2012;119(23):5429-5437.

26. Wang $Z$, et al. Integrin $\alpha 9$ depletion promotes $\beta$-catenin degradation to suppress triple-negative breast cancer tumor growth and metastasis. Int J Cancer. 2019;145(10):2767-2780.

27. Emori T, et al. Constitutive activation of integrin $\alpha 9$ augments self-directed hyperplastic and proinflammatory properties of fibroblast-like synoviocytes of rheumatoid arthritis. J Immunol. 2017;199(10):3427-3436.

28. Vlahakis NE, et al. The lymphangiogenic vascular endothelial growth factors VEGF-C and -D are ligands for the integrin $\alpha 9 \beta 1$. J Biol Chem. 2005;280(6):4544-4552.

29. Yokosaki Y, et al. The integrin alpha(9)beta(1) binds to a novel recognition sequence (SVVYGLR) in the thrombin-cleaved aminoterminal fragment of osteopontin. J Biol Chem. 1999;274(51):36328-36334.

30. Yokosaki Y, et al. Identification of the ligand binding site for the integrin alpha9 beta1 in the third fibronectin type III repeat of tenascin-C. J Biol Chem. 1998;273(19):11423-11428.

31. Hedin U, Thyberg J. Plasma fibronectin promotes modulation of arterial smooth-muscle cells from contractile to synthetic phenotype. Differentiation. 1987;33(3):239-246.

32. Wang K, et al. Prevention of intimal hyperplasia with recombinant soluble P-selectin glycoprotein ligand-immunoglobulin in the porcine coronary artery balloon injury model. J Am Coll Cardiol. 2001;38(2):577-582.

33. Noiseux N, et al. Bolus endovascular PDGFR-beta antisense treatment suppressed intimal hyperplasia in a rat carotid injury model. Circulation. 2000;102(11):1330-1336.

34. Bai $\mathrm{H}$, et al. Inhibition of programmed death-1 decreases neointimal hyperplasia after patch angioplasty. J Biomed Mater Res $B$ Appl Biomater. 2021;109(2):269-278

35. Tae HJ, et al. The N-glycoform of sRAGE is the key determinant for its therapeutic efficacy to attenuate injury-elicited arterial inflammation and neointimal growth. J Mol Med (Berl). 2013;91(12):1369-1381.

36. Liu W, et al. Toll-like receptor 2-deficiency on bone marrow-derived cells augments vascular healing of murine arterial lesions Life Sci. 2020;242:117189.

37. Huang XZ, et al. Fatal bilateral chylothorax in mice lacking the integrin alpha9beta1. Mol Cell Biol. 2000;20(14):5208-5215.

38. Muro AF, et al. Regulated splicing of the fibronectin EDA exon is essential for proper skin wound healing and normal lifespan. J Cell Biol. 2003;162(1):149-160.

39. Cai J, et al. HMGB1-driven inflammation and intimal hyperplasia after arterial injury involves cell-specific actions mediated by TLR4 Arterioscler Thromb Vasc Biol. 2015;35(12):2579-2593.

40. Li W, et al. The multifunctional Ca2+/calmodulin-dependent kinase II delta (CaMKIIdelta) controls neointima formation after carotid ligation and vascular smooth muscle cell proliferation through cell cycle regulation by p21. J Biol Chem. 2011;286(10):7990-7999.

41. Dhanesha N, et al. Targeting myeloid-specific integrin $\alpha 9 \beta 1$ improves short- and long-term stroke outcomes in murine models with preexisting comorbidities by limiting thrombosis and inflammation. Circ Res. 2020;126(12):1779-1794

42. Nakayama Y, et al. Blockade of interaction of alpha9 integrin with its ligands hinders the formation of granulation in cutaneous wound healing. Lab Invest. 2010;90(6):881-894.

43. Doddapattar P, et al. Fibronectin containing extra domain A induces plaque destabilization in the innominate artery of aged apolipoprotein E-deficient mice. Arterioscler Thromb Vasc Biol. 2018;38(3):500-508.

44. Jain M, et al. Gingerol inhibits serum-induced vascular smooth muscle cell proliferation and injury-induced neointimal hyperplasia by suppressing p38 MAPK activation. J Cardiovasc Pharmacol Ther. 2016;21(2):187-200.

45. Jain M, et al. Involvement of interleukin-1 receptor-associated kinase-1 in vascular smooth muscle cell proliferation and neointimal formation after rat carotid injury. Arterioscler Thromb Vasc Biol. 2015;35(6):1445-1455.

46. Khanna V, et al. Cholesterol diet withdrawal leads to an initial plaque instability and subsequent regression of accelerated iliac artery atherosclerosis in rabbits. PLoS One. 2013;8(10):e77037.

47. Ray JL, et al. Isolation of vascular smooth muscle cells from a single murine aorta. Methods Cell Sci. 2001;23(4):185-188

48. Kohan M, et al. EDA-containing cellular fibronectin induces fibroblast differentiation through binding to alpha4beta7 integrin 
receptor and MAPK/Erk 1/2-dependent signaling. FASEB J. 2010;24(11):4503-4512.

49. Jensen EC. Quantitative analysis of histological staining and fluorescence using ImageJ. Anat Rec (Hoboken). 2013;296(3):378-381.

50. Uemura Y, et al. Adipose-derived factor CTRP9 attenuates vascular smooth muscle cell proliferation and neointimal formation. FASEB J. 2013;27(1):25-33.

51. Cormier N, Yet al. Optimization of the wound scratch assay to detect changes in murine mesenchymal stromal cell migration after damage by soluble cigarette smoke extract. J Vis Exp. 2015(106):e53414.

52. Helmy IM, Azim AM. Efficacy of ImageJ in the assessment of apoptosis. Diagn Pathol. 2012;7:15.

53. Jain $\mathrm{M}$, et al. Intra-arterial drug and light delivery for photodynamic therapy using Visudyne: implication for atherosclerotic plaque treatment. Front Physiol. 2016;7:400. 